\title{
Bayesian Inference in the Modern Design of Experiments
}

\author{
Richard DeLoach ${ }^{*}$ \\ NASA Langley Research Center, Hampton, VA, 23681
}

\begin{abstract}
This paper provides an elementary tutorial overview of Bayesian inference and its potential for application in aerospace experimentation in general and wind tunnel testing in particular. Bayes' Theorem is reviewed and examples are provided to illustrate how it can be applied to objectively revise prior knowledge by incorporating insights subsequently obtained from additional observations, resulting in new (posterior) knowledge that combines information from both sources. A logical merger of Bayesian methods and certain aspects of Response Surface Modeling is explored. Specific applications to wind tunnel testing, computational code validation, and instrumentation calibration are discussed.
\end{abstract}

\section{Nomenclature}

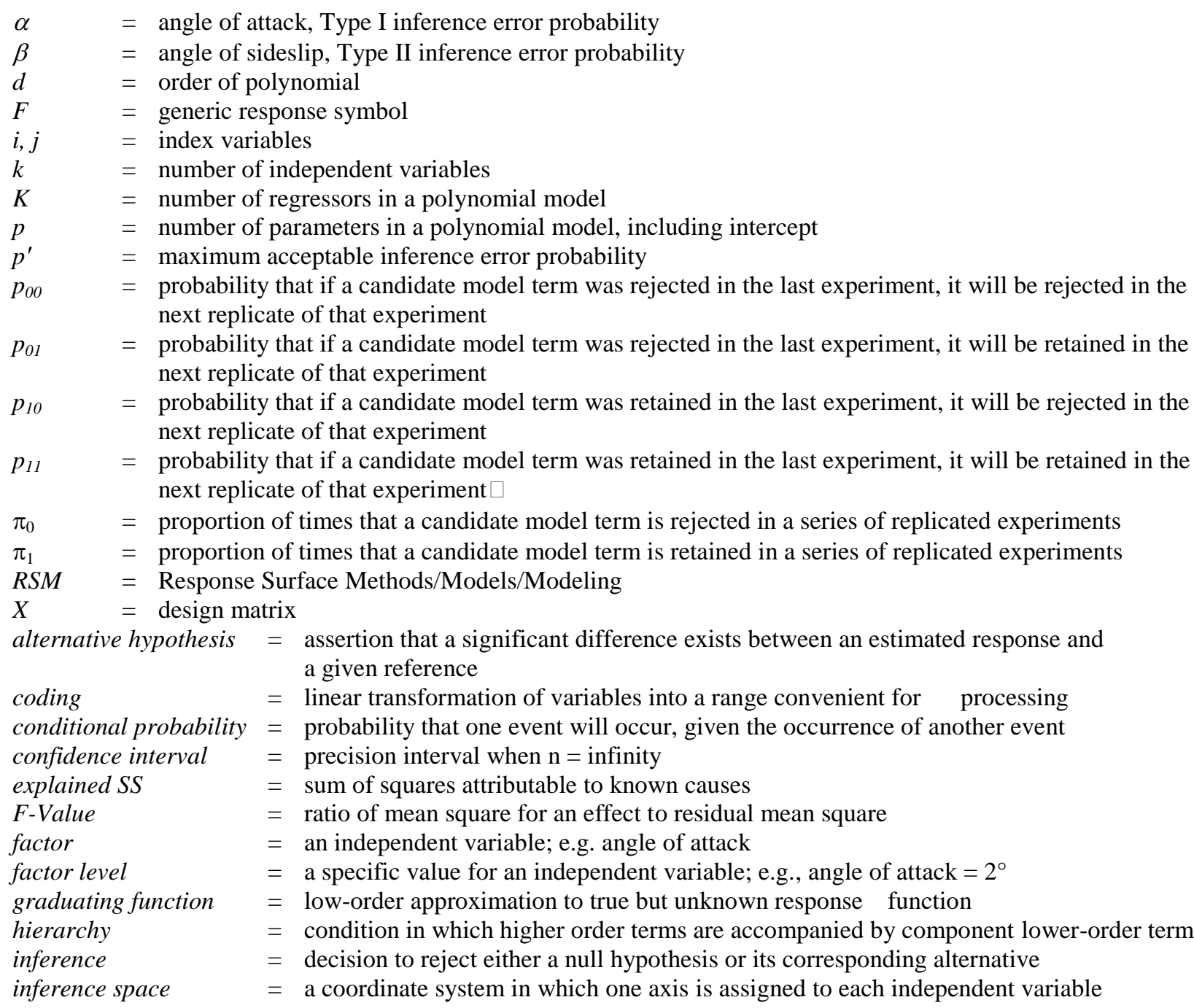

*Senior Research Scientist, NASA Langley Research Center, Hampton, VA. AIAA Senior Member. 
interaction effect

joint probability

LOF

main effect

marginal probability

MDOE

mean square

null hypothesis

OFAT

orthogonal

regressor

residual

residual mean square

residual SS

response surface model $=$

significance

site

t-limit

$t$-value

Type-I inference error $=$

Type-II inference error

$=$ change in effect due to change in factor level from low to high

$=$ probability of two events both occurring

$=$ lack of fit

$=$ change in response due to change in factor level from low to high

$=$ probability that an event will occur, independent of whether another event occurs

$=$ Modern Design of Experiments

$=$ ratio of sum of squares to degrees of freedom; variance

$=$ assertion than no difference exists between an estimated response and a given reference

$=$ One Factor At a Time

$=$ state in which regressors are all mutually independent

$=$ term in a regression model

$=$ difference between measurement and some reference

$=$ residual sum of squares divided by residual degrees of freedom

$=$ difference between total sum of squares and explained sum of squares

mathematical relationship between a response variable and multiple

independent variables

$=$ risk of erroneously rejecting a null hypothesis

$=\mathrm{a}$ point within an inference space representing some unique combination of factor levels

$=$ minimum acceptable signal-to-noise ratio

$=$ measured quantity expressed as multiple of standard error in measurement

erroneous rejection of a null hypothesis

erroneous rejection of an alternative hypothesis

\section{Introduction}

$I_{w}^{t}$ $\mathrm{t}$ is common to approach the analysis of information obtained in a response surface modeling experiment such as a wind tunnel test or a force balance calibration as if it stands alone, notwithstanding the fact that considerable prior information may exist on the subject under study in the experiment. A wind tunnel test may feature the replication of measurements acquired earlier on the same model, either in that facility or in another wind tunnel. An instrument undergoing a calibration may have been calibrated numerous times before. While it is not uncommon to compare current and prior results in such circumstances, such a comparison seldom extends beyond a subjective assessment that the agreement is generally satisfactory, or that it is sufficiently poor to be of concern. We do not usually exploit the fact that earlier experiments have yielded results that might be combined with our most recent findings to produce a composite outcome that reflects both the current and prior work.

There are several reasons to recommend such an integration of prior and current results when the opportunity presents itself to do so. By including prior data in the current analysis, the researcher avails himself of additional degrees of freedom that can reduce inference error risk in the current experiment and increase the precision with which results can be reported. That is to say, such a strategy has the potential to significantly reduce uncertainty, thereby improving the quality of the final result. Furthermore, these benefits can be obtained "for free," in that the expense of obtaining them has already been incurred, either by the researcher himself in a prior experiment or by some other research program entirely. In the former case, the merging of prior and current experimental findings results in an averaging down of the researcher's per-test costs. In the latter case, it results in a cost-effective leveraging of findings published in the literature or shared directly by colleagues who are able to do so.

An objective, systematic mechanism for merging current and prior experimental results is necessary to take advantage of this potential opportunity for quality improvement and average cost reduction. Fortunately, this mechanism is available in the form of Bayesian revision in statistical analysis. Bayesian revision can be applied to a statistical representation of the random variables comprising any experimental data sample to generate revised estimates for the location and dispersion metrics that characterize the probability distributions of such data, which reflect both prior and current information. This paper proposes the merger of such Bayesian methods with the response surface methods that are a key element of an integrated experiment design, execution, and analysis process known at NASA Langley Research Center as the Modern Design of Experiments.

The remainder of this paper is organized into five sections. Section II discusses the role of formal inference in the Modern Design of Experiments. Section III reviews Bayes' Theorem and gives examples of Bayesian inference. Section IV describes potential applications of Bayesian inference to response surface modeling experiments. Section 
$\mathrm{V}$ discusses certain aspects of Bayesian inference that distinguish it from conventional inference methods. Section VI provides some concluding remarks.

\section{The Role of Formal Inference in the Modern Design of Experiments}

The Modern Design of Experiments (MDOE) is an adaptation of industrial designed experiments that focuses on the special requirements of empirical aerospace research, including wind tunnel testing. ${ }^{1}$ It was first applied to wind tunnel testing at NASA Langley Research Center in 1997 and has since been used in over 100 major experiments as a means of delivering higher quality and greater productivity than conventional one factor at a time (OFAT) testing methods used traditionally in experimental aeronautics. Differences between MDOE and OFAT methods for wind tunnel testing stem primarily from contrasting views of the objective of this activity. Colloquially stated, conventional OFAT practitioners conduct wind tunnel tests to acquire data, while MDOE practitioners conduct wind tunnel tests to acquire knowledge. That is, the OFAT practitioner's typical test strategy is to directly measure system responses such as forces and moments for as many independent variable (factor) combinations of interest as resource constraints will allow, while the MDOE practitioner's typical strategy is to make some relatively small number of measurements that is nonetheless ample to develop a mathematical model that can adequately predict responses for all factor combinations of interest, and to make no more measurements than that.

MDOE predictions are based on response surface models fitted by regression or other means from a sample of experimental data. The size of this sample is minimized to reduce direct operating cost and cycle time as noted, and the selection and acquisition order of the individual data points are optimized to reduce uncertainty in predictions made from models that are fitted from the data. ${ }^{2,3}$

Fitting a regression model to a relatively small data sample offers certain advantages over the traditional OFAT exhaustive enumeration strategy that requires every interesting combination of independent variable levels to be physically set. Besides the obvious cost and cycle-time reductions achievable by minimizing data volume, the modeling approach enables interpolation, so that response estimates can be made for many other factor combinations than those physically set in the test.

Low-order polynomials are a convenient and common form of response model fitted from experimental data. Such a model can be regarded as a truncated Taylor series representation of the true but unknown functional relationship between some response of interest (a force or moment, say), and the independent variables that influence that response (angle of attack, Mach number, control surface deflections, etc). Often such models are fitted over a limited range of the independent variables so that the resulting model—called a graduating function — can be regarded as a mathematical "French curve" that fits the data adequately in this range. Other models are then developed for other ranges of the variables. By restricting the range of the independent variables sufficiently, an adequate fit to the data can be secured for arbitrarily low-order graduating functions. Typically we seek a reasonable compromise between the order of the fitting model and the range of independent variables to be fitted.

Progress is made in an MDOE experiment through an iterative process in which a proposed model is subjected to criticism intended to test certain assumptions upon which the validity of the model rests. Typically this criticism takes the form of various tests applied to model residuals, which can be expected to contain no information for a well-fitted model, and which reveal trends and other information when the model is an inadequate representation of the underlying data. Likewise, certain distributional assumptions of the residuals are commonly tested.

Information revealed in an analysis of the residuals may suggest improvements to the model, or the need to acquire additional data to fit a more complex function of the independent variables. A revised model is then produced, which is again subjected to criticism. This process continues until the researcher is satisfied that the model is adequate for a particular purpose defined during the design of the experiment. In a wind tunnel test, that purpose typically is to predict measured responses within a specified tolerance and with an acceptable level of confidence. The tolerances and confidence levels are documented as part of the MDOE experiment design process.

A polynomial response model is structured as a linear sum of monomials, each consisting of the product of a numerical regression coefficient and some function of the independent variables. Suppose, for example, that lift measurements have been made over a sufficiently truncated range of angle of attack, $x_{1}$, and angle of sideslip, $x_{2}$, that we can reasonably assume that a $2^{\text {nd }}$-order polynomial would be adequate to represent the coefficient of lift as a function of these two variables over this range. We would then postulate the following model:

$$
y=b_{0}+b_{1} x_{1}+b_{2} x_{2}+b_{12} x_{1} x_{2}+b_{11} x_{1}^{2}+b_{22} x_{2}^{2}
$$

where $y$ represents the coefficient of lift and the $b_{i}$ are numerical coefficients calculated from the data by regression methods. 
Regression is simply a defined computational process that will generate a set numerical regression coefficients for this model without regard for physical reality. For example, if there is no interaction between $x_{1}$ and $x_{2}$ (that is, if the effect on $y$ of a given change in $x_{1}$ is the same no matter the value of $x_{2}$ ), then the $x_{1} x_{2}$ cross term would be superfluous and the true value of the $b_{12}$ coefficient in this model would be zero. Unfortunately, unexplained variance present in every experimental data sample results in uncertainty in the estimates of regression coefficients. The result is that, except for sheer coincidence, the fitted coefficients of each of the six terms in a second-order polynomial function of two independent variables will be non-zero, even if the physical phenomenon represented by a given term does not occur in nature.

The researcher is motivated to minimize the number of terms in a response surface model by dropping all terms for which the true value of the regression coefficient is zero. This is so for a number of reasons. One is that since each term in the model carries some uncertainty due to the experimental error in its regression coefficient, the smaller the number of such imperfect terms that are summed to form a response estimate, the smaller the uncertainty will be in that estimate. Indeed, it can be shown ${ }^{4}$ that the prediction variance averaged across all points used to generate a well-fitted regression model is simply

$$
\overline{\operatorname{Var}(y)}=\frac{p \sigma^{2}}{n}
$$

where $p$ is the number of terms in the model including the intercept (so six for the present model) and $n$ is the number of points in the data sample used to generate the model. We note in passing that $n \geq p$ is a condition for fitting a model so that in the limiting case for which $n=p$ and there are just enough points to fit the model, the average model prediction variance is just $\sigma^{2}$, the intrinsic variance in the data. However, in the more general case in which $n>p$ so that there are residual degrees of freedom available, the average prediction variance is less than the intrinsic variance of the data. This is an additional argument in favor of response surface methods for estimating system responses, rather than the individual measurements that characterize OFAT testing.

Beyond reducing model prediction variance, the researcher is motivated to develop compact response models in order to clarify the underlying physics. In the example already cited, if there is no physical interaction between the two independent variables in Eq. (1), then retaining such an interaction term in the model simply adds unnecessary clutter.

The process of response surface model building reduces to a series of decisions, or inferences, with respect to whether the regression coefficient of a given candidate term in a proposed model conveys information about the true system response, or whether it is due to nothing more than experimental error. Formally, we establish a null hypothesis for each model term except the intercept, declaring it to be superfluous. We only reject this null hypothesis (and therefore retain the candidate term in the model) if there is compelling objective evidence to do so.

The decision of whether or not to reject the null hypothesis for a given term in a proposed model is made with the aid of a reference probability distribution. This distribution reflects the dispersion in experimental estimates of the regression coefficient, and is therefore related to the "noise" in an experimental result. We reject the null hypothesis only when the magnitude of the estimated regression coefficient is sufficiently different from zero that we can infer that it is not zero with an acceptably low probability of an inference error. Stated another way, we demand a certain minimum signal-to-noise ratio as a condition for rejecting the null hypothesis.

Reference distributions are illustrated in Fig. 1 for two regression coefficients. We generally appeal to the Central Limit Theorem to support an assumption that these distributions are Gaussian. If we infer that the true value of the $i^{t h}$ regression coefficient is indeed greater than zero, then the probability of an error in this inference, $p_{i}$, is

$$
p_{i}=\frac{1}{\sigma_{i} \sqrt{2 \pi}} \int_{-\infty}^{0} \exp \left[-\frac{\left(x-b_{i}\right)^{2}}{2 \sigma_{i}^{2}}\right] d x
$$

which is simply the area under the probability density function to the left of zero, where $\sigma_{\mathrm{i}}$ is the standard deviation of this distribution. Obviously, the further from zero that $b_{i}$ is, the smaller is the probability, $p_{i}$, of making an inference error by rejecting the null hypothesis. Such an error would result in retaining a non-existent term in the response model, and is known as a Type I inference error. For example, if in Eq. (1) we were to retain the $b_{12} x_{1} x_{2}$ term when there was in fact no physical interaction between $x_{1}$ and $x_{2}$, then we would have made this type of inference error. 


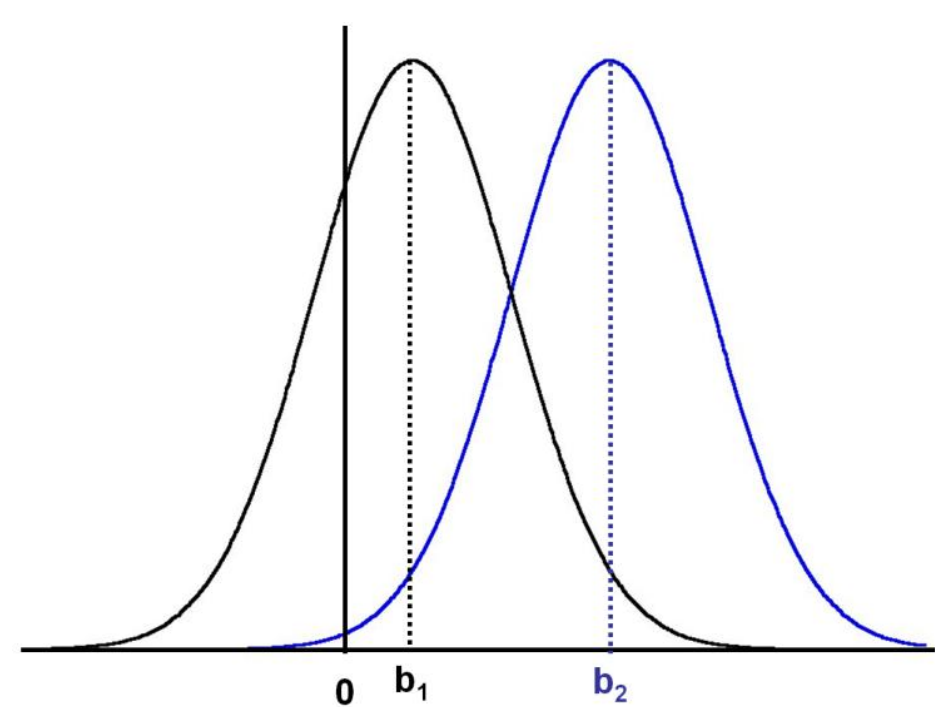

Figure 1. The magnitude of two regression coefficients, showing dispersion in their experimental estimates (reference distributions). We reject the null hypothesis for $b_{2}$ but not for $b_{1}$. Higher-order columns are generated from the first order columns via term-by-term manipulations of the lower-order terms. For example, the elements of the $x_{1} x_{2}$ column are generated by simply multiplying the $x_{1}$ and $x_{2}$ levels for each data point. Likewise, columns for quadratic terms are generated by squaring the corresponding elements in the first-order columns, and so on. Before the design matrix is generated the variables are commonly scaled and centered by a linear transformation that maps them into a range from -1 to +1 , with " 0 " corresponding to the center of the range. This minimizes certain round-off errors that can occur when the numerical magnitudes of different variables in physical units are considerably different (angle of attack on the order of ones to tens and Reynolds numbers in the range of millions, for example).

The reader is referred to standard texts on regression analysis and response surface modeling ${ }^{4-7}$ for additional details on the construction of the design matrix, $\mathbf{X}$. Suffice it to say that two points are key with respect to the design matrix: 1) for a given measurement environment, the regression coefficients and their standard errors are completely defined by the design matrix and the resulting vectors of measured responses, which means that inference errors are directly impacted by the nature of the design matrix, and 2) the structure of the design matrix is defined by the researcher prior to the test. This is the reason that there is so much potential to influence the quality and productivity of a wind tunnel test or any other type of experiment by the design of the experiment.

A $1 \times p$ vector of regression coefficients, $\mathbf{b}$, can be computed directly from the $p \times n$ design matrix, $\mathbf{X}$, and the $1 \times n$ vector of corresponding response measurements, $\mathbf{Y}$, where $p$ is the number of terms in the fitted model including the intercept term as before, and $n$ is the number of points used to fit the response model:

$$
\mathbf{b}=\left(\mathbf{X}^{\prime} \mathbf{X}\right)^{-1} \mathbf{X}^{\prime} \mathbf{Y}
$$

The standard error for each regression coefficient comes from the covariance matrix, $\mathbf{C}$, computed by inverting the product of the transpose of the design matrix $\left(\mathbf{X}^{\prime}\right)$ and the design matrix, $\mathbf{X}$, then multiplying every term by $\sigma^{2}$, the unexplained variance in the response measurements.

$$
\mathbf{C}=\left(\mathbf{X}^{\prime} \mathbf{X}\right)^{-1} \sigma^{2}
$$

The covariance matrix is a $p \times p$ square matrix. The standard error for the $i^{\text {th }}$ regression coefficient is simply the square root of the $i i^{\text {th }}$ (diagonal) element of this matrix. These represent the standard deviations of the reference 
distributions used to infer whether or not to reject the null hypothesis that a given candidate term in the model should be eliminated. See Fig. 1.

This discussion has highlighted the role that probability and statistical inference plays in the response surface methods used in an MDOE analysis. Inference error probabilities are estimated for each candidate regression coefficient via Eqs. (3), (4), and (5), and compared with inference error criteria developed in the experiment design phase. By one common convention, the null hypothesis is only rejected if the corresponding probability of an inference error is no greater than 0.05 . By this criterion, we require the estimated regression coefficient's signal to noise ratio to be large enough that we can say with $95 \%$ confidence that it is not zero, and that the corresponding term does therefore belong in the regression model. Coefficients with a smaller signal to noise ratio may in fact be non-zero, but those terms are rejected from the model on the grounds that the coefficients are not large enough for us to say with confidence that they belong.

Table 1 describes a response surface model generated in a recent wind tunnel test at Langley Research Center. This model represents lift coefficient as a function of the angles of attack and sideslip over ranges of those variables that were sufficiently constrained that it was believed a good fit could be achieved with a full second-order response model in the two independent variables.

Table 1. Initial Regression Model.

\begin{tabular}{|r|c|c|c|}
\hline \multicolumn{1}{|c|}{ Factor } & Regression Coefficient & $\begin{array}{c}\text { Std Err of } \\
\text { Coefficient }\end{array}$ & $\begin{array}{c}\text { Inference Error Probability } \\
\text { if Null Hypothesis Rejected }\end{array}$ \\
\hline Intercept & 0.5389 & $5.48 \mathrm{E}-04$ & -- \\
\hline$x_{1}$, Angle of Attack & $9.099 \mathrm{E}-02$ & $5.48 \mathrm{E}-04$ & $<0.0001$ \\
\hline$x_{2}$, Angle of Sideslip & $1.967 \mathrm{E}-03$ & $5.48 \mathrm{E}-04$ & 0.0050 \\
\hline$x_{1}^{2}$ & $-1.051 \mathrm{E}-03$ & $5.48 \mathrm{E}-04$ & 0.0842 \\
\hline$x_{2}^{2}$ & $3.188 \mathrm{E}-03$ & $5.48 \mathrm{E}-04$ & 0.0002 \\
\hline$x_{1} x_{2}$ & $6.35 \mathrm{E}-05$ & $7.75 \mathrm{E}-04$ & 0.9363 \\
\hline
\end{tabular}

Recall that we progress in an MDOE analysis by postulating a candidate model and then subjecting it to criticism. We conduct a critical examination of the candidate model with the intent of identifying ways to improve it. In the case of the lift model represented in Table 1, we see that if we reject the null hypothesis for the first-order angle of attack term, the error probability associated with that inference is negligible $(<0.0001)$. We conclude that we would be very unlikely to improve the model (and would therefore be at some risk of degrading it) if we failed to include the first-order angle of attack term in the model. This is consistent with experience and subject matter expertise, from which we realize that pre-stall lift is very nearly a linear function of angle of attack. Likewise, decisions to retain the first- and second-order sideslip angle terms seem to be well justified. However, two of the five regressors have inference error probabilities that exceed our 0.05 criterion for rejecting the null hypothesis and retaining the term. These are the pure quadratic angle of attack term with an inference error probability of 0.0842 , and the interaction term with an inference error probability of 0.9363 .

We interpret these probabilities as descriptive of the chance that a coefficient of the size estimated from the data could have been computed strictly as the result of experimental error. This probability is especially high for the interaction term, reflecting the fact that our estimate of the coefficient for that term is two orders of magnitude smaller than the smallest coefficient for any other regressor in the model and only represents 0.08 of one standard deviation, so a very small signal-to-noise ratio. We therefore tentatively drop both of these terms to produce a reduced model of the following form

$$
y=b_{0}+b_{1} x_{1}+b_{2} x_{2}+b_{22} x_{2}^{2}
$$

By Eq. (2), reducing the parameter count, $p$, from six to four will have reduced that component of the prediction variance due to random error by a third. However, if eliminating one or both of these two terms ultimately proves to 
be unjustified, this decision will result in a systematic divergence between predicted and measured lift estimates. If this is the case and the resulting increase in systematic prediction error over the range of independent variables tested is greater than the decrease in random prediction error achieved by reducing the parameter count, then the decision to eliminate these terms will have been an improper one.

Again, we must subject the revised model to criticism, seeking to identify weaknesses that would render it inadequate for its purpose, which is to predict measured lift values over a specified range of independent variables within an acceptable tolerance. The iterative process by which the results of a proposed model are subjected to criticism, revised, and retested, continues until no rationale can be found to reject the model.

The model in Eq. (6) was tested by using it to predict lift coefficients for 16 combinations of angle of attack and sideslip angle within the ranges tested. Measurements were made for those points, and the residuals - differences between corresponding measured and predicted lift values - were computed. The average residual magnitude for lift coefficient was 0.0013 , which indicates a relatively high accuracy for the model. However, the accuracy requirement for this particular test was 0.0010 , indicating that the predictions associated with the response model of Eq. (6) were out of tolerance by about $30 \%$. We conclude that the model is still inadequate and consider other ways to improve it.

We re-examine the reduced model in Eq. (6) by reviewing the inferences that resulted in decisions to drop or retain individual terms from the full second-order model of Eq. (1). Table 1 leaves little doubt that there is no interaction between the angles of attack and sideslip for this particular vehicle over the model attitude ranges that were tested. The decision to drop the $x_{1} x_{2}$ term therefore seems sound (although a review of individual data points to look for recording errors or other evidence of unexplained behavior is customary). The decision to drop the pure quadratic angle of attack term is not quite as easy to defend. The inference error risk tolerance level was somewhat arbitrarily chosen to be 0.05 , implying a requirement for $95 \%$ confidence in a decision to retain each term in the model. Table 1 shows that the estimated inference error probability was only slightly greater than 0.08 , close to the 0.05 cut-off and presumably suggesting that we could retain the quadratic term with almost $92 \%$ confidence in our decision to do so. Given the somewhat arbitrary nature of the declared risk tolerance criterion, it is reasonable to revisit the question of retaining the quadratic angle of attack term in the model. If it is legitimate to do so, then retaining the extra term would allow us to explain that much more of the total variance in the data, resulting in a corresponding reduction in the unexplained variance. A relatively small reduction in the unexplained variance could be sufficient to reduce the average magnitude of our lift coefficient residuals by the 0.0003 amount necessary to bring us into tolerance.

There is an additional, perhaps even more important reason to revisit the decision to declare the quadratic angle of attack term insignificant in this case. The purpose of this analysis was to examine whether the regression coefficient for the pure quadratic angle of attack term could be used as an objective and sensitive indicator of the onset of stall. The intent was to correlate this coefficient with various configuration changes that had the potential to influence stall. A significant quadratic angle of attack term suggests the beginning of the slope reduction for lift that presages the onset of stall.

Various alternatives are available. An easy alternative that is not recommended is to simply revise our inference error risk tolerance in light of developments, declaring now that we require only $90 \%$ confidence, say, before we decide to retain any given term in the model. This would ensure that the 0.08 inference error probability associated with the decision to reject the null hypothesis for the quadratic term would represent an acceptable risk, and we could therefore retain the term and proceed. This practice of "moving the goal posts" is undesirable because it forecloses options we would otherwise have for entirely objective inferences. It is also dangerous because it provides a mechanism whereby our inevitable prejudices can influence the result of an experiment. Despite the best of intentions to remain neutral and objective, the fact is that there will be circumstances when we might prefer that the true model have a quadratic angle of attack term - perhaps to comport with a theory we have formulated or to be consistent with other data we have reported. There might be other times when this indicator of the onset of stall might be inconvenient. If we are permitted to adjust the inference error risk tolerance arbitrarily, it is too easy to influence the results in a direction that we might prefer, even if only subconsciously.

Another alternative is to acquire additional data. The added data will increase precision and make it clearer whether the coefficient for the quadratic angle of attack term is large enough to be distinguished from zero with at least $95 \%$ confidence. This alternative can be inconvenient if the test has already been completed, and in any case it would require additional cost and cycle time.

We now consider how to exploit a situation in which other experimental results are available to us. If the vehicle under investigation has been tested before - perhaps in another wind tunnel—and a similar response surface model has been developed and documented, it would be convenient if we could objectively combine the two results. Note that we might be hesitant to simply combine the raw data from the other test with ours. Between-tunnel differences could be attributable to such factors as differential corrections for wall effects due to a difference in methodology or 
a difference in test section geometry, differences in instrumentation, differences in the fidelity of the model, etc. Such between-tunnel differences have the potential to add enough additional unexplained variance to an analysis of both sets of data combined, that the subtle effects we seek to illuminate could be lost in the ensuing noise.

As an alternative, consider a case in which the first experiment has been subjected to a response surface analysis, and inferences have been made about whether to retain or reject terms in the response model for lift coefficient developed in that model. We describe in the next section how an application of Bayes' Theorem can objectively combine the results of two such experiments.

\section{Bayes' Theorem and Bayesian Inference}

We adopt a standard notation by using $\mathrm{P}(\mathrm{A} \mid \mathrm{B})$ to represent the conditional probability that event " $\mathrm{A}$ " will occur, given that event " $\mathrm{B}$ " has occurred. We say that the probability of " $\mathrm{A}$ " is conditional on " $\mathrm{B}$ " in this case. For example, event " $\mathrm{A}$ " might represent a regression coefficient in a candidate response model being non-zero. Event "B" might be the acquisition of some sample of data. In that case, $\mathrm{P}(\mathrm{A} \mid \mathrm{B})$ represents the probability that the coefficient is significant, given the data sample.

In general, $\mathrm{P}(\mathrm{A} \mid \mathrm{B})$ is not the same as $\mathrm{P}(\mathrm{B} \mid \mathrm{A})$; however, they are related to each other through a relatively simple relationship comprising Bayes' Theorem. Bayes' Theorem can be exploited to objectively revise early conclusions in light of new evidence, as will be shown in this section. For example, we originally concluded in the previous section that the pure quadratic angle of attack term in a candidate model for lift coefficient could be rejected, and we have now postulated additional evidence in the form of a second wind tunnel test. The question is, does the additional evidence indicate that we should revise our original belief about the significance (or lack thereof) of the quadratic angle of attack term for this model? To address this question, we review Bayes' Theorem and give some elementary examples of how it can be used.

\section{A. Derivation of Bayes' Theorem}

Bayes' Theorem is easily derived from the definition of conditional probability, as expressed in terms of a joint probability. The joint probability of events $\mathrm{A}$ and $\mathrm{B}$ is represented as $\mathrm{P}(\mathrm{A} \cap \mathrm{B})$ and defined as the probability that events A and B both occur. We define the prior probability of event "B," written as $\mathrm{P}(\mathrm{B})$, as the probability that " $\mathrm{B}$ " will occur independent of whether event "A" occurs. We can now express both the conditional probability of "A" given "B," and the conditional probability of "B" given " $\mathrm{A}$ " in terms of their joint and prior probabilities, as follows:

$$
\begin{aligned}
& P(A \mid B)=\frac{P(A \cap B)}{P(B)} \\
& P(B \mid A)=\frac{P(A \cap B)}{P(A)}
\end{aligned}
$$

From Eqs. (7) we have

$$
P(A \cap B)=P(A \mid B) P(B)=P(B \mid A) P(A)
$$

which is the well-known product rule for probabilities. It leads directly to Bayes' Theorem:

$$
P(A \mid B)=\frac{P(B \mid A) P(A)}{P(B)}
$$

The quantity $\mathrm{P}(\mathrm{B} \mid \mathrm{A}) / \mathrm{P}(\mathrm{B})$ is often described as the normalized likelihood function. We say, then, that the conditional probability of "A" given "B" is just the prior probability of "A" times this normalized likelihood function. The importance of the likelihood function in a Bayesian analysis derives from the fact that it depends on " $\mathrm{B}$ ", and thus represents the mechanism by which the prior probability of " $\mathrm{A}$ " is modified by the observation of "B." 


\section{B. Illustration of Basic Concepts}

We can illustrate Bayes' Theorem with the following example. Suppose we are presented with a coin that may be fair (equal probability of heads and tails) or may be weighted in such a way that the probability of tails is twice the probability of heads. We have no prior information about the coin so the proposition that it is fair is equally likely as the proposition it is weighted, based on the information at our disposal. We therefore decide to toss the coin 100 times to test the hypothesis that the coin is fair, and we find that we get heads 43 times and tails 57 times. Were we flipping a fair coin or a loaded one?

Note that the fact that we did not get 50 heads does not argue against the coin being fair. For 100 tosses of a fair coin we will get 50 heads more often than any other number, but the probability of getting precisely 50 heads is actually rather low-less than 0.08 . So even with a fair coin we would expect to get something other than 50 heads over $92 \%$ of the time.

The loaded coin is more likely to come up tails than heads on any one toss and so we would expect more tails than heads if the coin is loaded. In fact, we got 57 tails and only 43 heads. If you think this evidence favors an inference that the coin is weighted because there were so many more tails than heads, you should be willing to give odds in a wager that this is so. Given the evidence you have, what odds would you be willing to offer the author to entice him to bet against you in a million-dollar wager in which you win if the coin is loaded and he wins if it is fair? Stated another way, if prior to the test there was no reason to believe the coin was either fair or weighted, and given that the coin came up heads only 43 times in 100 tosses, what is the probability that the coin is fair?

We can use Bayes' Theorem to compute this probability by letting " $A$ " represent the event that the coin is fair and letting " $\mathrm{B}$ " represent the event that we observe 43 heads in 100 tosses. Then $\mathrm{P}(\mathrm{A} \mid \mathrm{B})$ is the conditional probability that the coin is fair, given that 43 heads were observed in 100 tosses, which is the probability we wish to compute.

The quantity $\mathrm{P}(\mathrm{B} \mid \mathrm{A})$ is simply the probability of getting 43 heads in 100 tosses if the coin is fair. This is easy to calculate from the binomial probability formula that gives the probability of " $\mathrm{x}$ " successes in $\mathrm{N}$ trials, given that the probability of success in any one trial is $p$ :

$$
P(B \mid A)=\frac{N !}{x !(N-x) !} p^{x}(1-p)^{N-x}
$$

For $N=100, x=43$, and $p=0.5, \mathrm{P}(\mathrm{B} \mid \mathrm{A})=0.0301$ by this formula.

$\mathrm{P}(\mathrm{A})$ is the prior probability that the coin is fair. That is, this is the probability that the coin is fair before we obtain any additional evidence in the form of coin-toss test results. Since we had no reason to suspect the coin was either fair or weighted before the test, $\mathrm{P}(\mathrm{A})=0.5$.

For computational convenience in a problem like this, we re-cast $\mathrm{P}(\mathrm{B})$ in Eq. (9) by first noting that

$$
P(B)=P(A \cap B)+P(\bar{A} \cap B)
$$

where the bar over "A" implies "not A". So Eq. (11) simply states that the probability of "B" independent of "A" is the probability of "B" when " $A$ " occurs plus the probability of " $B$ " when " $A$ " does not occur. From Eq. (7b) with obvious extensions to the "not A" case, Eq. (11) becomes

$$
P(B)=P(B \mid A) P(A)+P(B \mid \bar{A}) P(\bar{A})
$$

and Bayes' Theorem as expressed in Eq. (9) becomes

$$
P(A \mid B)=\frac{P(B \mid A) P(A)}{P(B \mid A) P(A)+P(B \mid \bar{A}) P(\bar{A})}
$$

For the current example, $\mathrm{P}(\overline{\mathrm{A}})$ is simply the prior probability that the coin is not fair, which is 0.5 . The quantity $\mathrm{P}(\mathrm{B} \mid \overline{\mathrm{A}})$ is the probability that we would observe 43 heads in 100 tosses given that the coin was not fair; that is, if it was weighted to produce twice as many tails as heads. In that case the probability of a head is $1 / 3$ rather than 0.5 , 
and we can use Eq. (10) with $N=100, x=43$, and $p=1 / 3$ to determine that $\mathrm{P}(\mathrm{B} \mid \overline{\mathrm{A}})=0.0107$. Inserting all calculations into Eq. (13) we find

$$
P(A \mid B)=\frac{(0.0301)(0.5)}{(0.0301)(0.5)+(0.0107)(0.5)}=0.7377
$$

So contrary to intuitive expectations resulting from the fact that there were several more tails than heads, we conclude that the coin is probably not weighted to favor tails by two to one, and in fact there are roughly three chances in four that the coin is fair. That is, it is roughly three times more likely that the coin is fair than that it is weighted to favor tails. This is because the probability of observing 43 heads in 100 tosses of a fair coin (0.0301) is roughly three times the probability of observing 43 heads in 100 tosses of a coin weighted to make it twice as likely to see a tail as a head on any one toss. Putting it another way, there simply were not enough tails observed to support the hypothesis that tails were twice as likely as heads. We see that the acquisition of additional empirical evidence has resulted in a revision of the probability we originally assigned to the coin being fair, from 0.5000 to 0.7377 . To use the language of Bayesian revision, we say that the prior probability was 0.5000 , while the posterior probability is 0.7377 .

To return to our initial question, you should not offer any odds to entice the author to bet a million dollars with you that the coin is fair, based on this evidence. On the contrary, the author would still have a substantial advantage if he took the bet that the coin was fair and offered you, say, 2:1 odds to bet that it was weighted.

\section{An Application to Scientific Research}

The above example was contrived to illustrate how the basic concepts of conditional, joint, and prior probability can be combined to revise an earlier opinion based on new evidence via Bayes' Theorem. We now present an example first offered by Ronald Fisher, ${ }^{8}$ to illustrate the application of Bayesian revision in a scientific investigation in which new empirical evidence is introduced that conflicts with prior conclusions.

Fisher considered a genetics experiment involving three types of mice. One type inherits a dominant gene related to fur color from both parents (designated here as "CC"), one type inherits the recessive gene for color from both parents ("cc"), and one type inherits one dominant color gene and one recessive color gene (Cc). Any mouse of this particular species inheriting the dominant color gene is black (so both the $\mathrm{CC}$ and Cc types). A mouse inheriting two recessive color genes (cc) is brown. The probability that parent mice of a particular gene composition will produce offspring of a given genetic composition is well known from genetic theory and summarized in Table 2 .

Table 2. Probabilities of Genetic Composition of Offspring in Mice.

\begin{tabular}{|c|c|c|c|}
\hline \multirow{2}{*}{ Parents } & \multicolumn{3}{|c|}{ Offspring } \\
\cline { 2 - 4 } & CC (black) & Cc (black) & cc (brown) \\
\hline $\mathrm{CC}+\mathrm{cc}$ & 0 & 1 & 0 \\
\hline $\mathrm{Cc}+\mathrm{cc}$ & 0 & $1 / 2$ & $1 / 2$ \\
\hline $\mathrm{Cc}+\mathrm{Cc}$ & $1 / 4$ & $1 / 2$ & $1 / 4$ \\
\hline
\end{tabular}

These probabilities in Table 2 are easily established by considering two fair coins that are tossed, one representing the father and one the mother. Heads means the parent contributes the first gene of its " $x \mathrm{x}$ " pair and tails means it contributes the second. The first row in Table 2 shows that the offspring for those particular parents can never receive two dominant color genes or two recessive color genes because one parent can only contribute one type and the other parent can only contribute the other. So the probability of an offspring with one gene of each type is $100 \%$, with no chance of producing an offspring with either two dominant or two recessive genes.

Likewise, the second row in the table corresponds to the case in which one of the parents cannot contribute a dominant color gene, so the probability of an offspring with two dominant genes is zero. There is a $100 \%$ probability that the offspring will have one recessive gene since that is all that one parent has to offer, but there is an equal probability that it will inherit the other parent's dominant or recessive gene. Finally, the last row in the table describes the case when both parents have both genes, in which case the probability that the offspring will have two dominant genes is the probability of getting two "heads" with two tosses of a fair coin (1/4), and likewise the 
probability of two recessive genes is also $1 / 4$. There is a probability of $1 / 2$ that the offspring will inherit one gene of each kind.

Mice with two dominant color genes are called homozygotes while those with one dominant gene and one recessive gene are called heterozygotes. Assume we have a black mouse that is the offspring of two heterozygotes (last line in Table 2). Clearly, the prior probability that this black mouse is a homozygote is $1 / 3$ and the prior probability that it is a heterozygote is $2 / 3$. So absent any other information, we would say it is likely (2:1 odds) that the mouse has both color genes.

Now suppose, as Fisher did, that we avail ourselves of additional evidence in the form of an experiment in which we mate our black mouse with a brown one, known to have two recessive color genes. Fisher assumed that the result of such an experiment was the production of seven black offspring. We wish to compute, as Fisher did, the posterior (post-test) probability that the test mouse had both a dominant and a recessive color gene. In this case, we let "A" correspond to the test mouse having both types of color gene and we let " $\mathrm{B}$ " correspond to the test result that seven black mice were produced by mating it with a brown mouse. We wish to compute $P(A \mid B)$ - the probability that the test mouse has both types of gene, given that mating with a brown mouse produced seven black offspring.

In this case, $P(A)$ is $2 / 3$, from the data in the table, and $P(\bar{A})$ is $1 / 3$. Also from the table, the probability of producing a single black offspring from a $\mathrm{Cc}+\mathrm{cc}$ pairing is $1 / 2$, so $P(B \mid A)$ is just $(1 / 2)^{7}$. We know from its color and pedigree that if the black test mouse does not have both color genes, it has to have two dominant genes, in which case its offspring will always be black. Therefore, we know that $P(B \mid \bar{A})$ is 1 . Inserting these values for prior and conditional probabilities into Eq. (13) yields this:

$$
P(A \mid B)=\frac{(1 / 2)^{7}(2 / 3)}{(1 / 2)^{7}(2 / 3)+(1)(1 / 3)}=\frac{(1 / 2)^{6}}{1+(1 / 2)^{6}}=\frac{1}{65}
$$

This example illustrates how dramatically prior knowledge can be altered by additional information. In this case, we thought before the experiment that the odds of the black test mouse having both color genes was 2:1 in favor of that proposition. This initial probability estimate was based on prior knowledge that each parent had a dominant and a recessive color gene, but when that prior knowledge was augmented with additional information from the mating experiment, the odds that the test mouse had both gene types changed from 2:1 in favor to 64:1 against, a substantial change in opinion.

It is instructive to represent the litter of seven new-born mice as a sequence of independent "data points" to examine how our perceptions would have changed with each new birth. Applying Bayes' Theorem sequentially, the conditional probability that the black test mouse had a recessive color gene given the evidence would have diminished with each new birth. The corresponding probability that the test mouse possessed two dominant color genes would have grown with each new birth, as in Table 3.

Table 3. Probability that male parent mouse has two dominant color genes, given that a) the female parent has two recessive color genes, and b) a series of black offspring are born.

\begin{tabular}{|c|c|}
\hline New-Born Black Mice & Probability that father is CC \\
\hline 0 & $1 / 3$ \\
\hline 1 & $1 / 2$ \\
\hline 2 & $2 / 3$ \\
\hline 3 & $4 / 5$ \\
\hline 4 & $8 / 9$ \\
\hline 5 & $16 / 17$ \\
\hline 6 & $32 / 33$ \\
\hline 7 & $64 / 65$ \\
\hline
\end{tabular}


The data in Table 3 are plotted in Fig. 2. Note that our confidence that the test mouse has two dominant genes grows with each new data point in this case, but approaches $100 \%$ only asymptotically. That is, no matter how much evidence we acquire, there will always be some prospect, however remote, that the results are due simply to chance. Figure 2 illustrates a general aspect of inference on the basis of data with uncertainty, which is that we can never be absolutely sure of an inference, no matter how great the volume of evidence supporting it. This principle can be exploited to control the cost of experimentation, by adopting a policy whereby the cost of acquiring additional data is no longer borne once the inference error probability drops below some prescribed threshold, or equivalently, once confidence in a given inference exceeds a specified threshold (95\%, say).

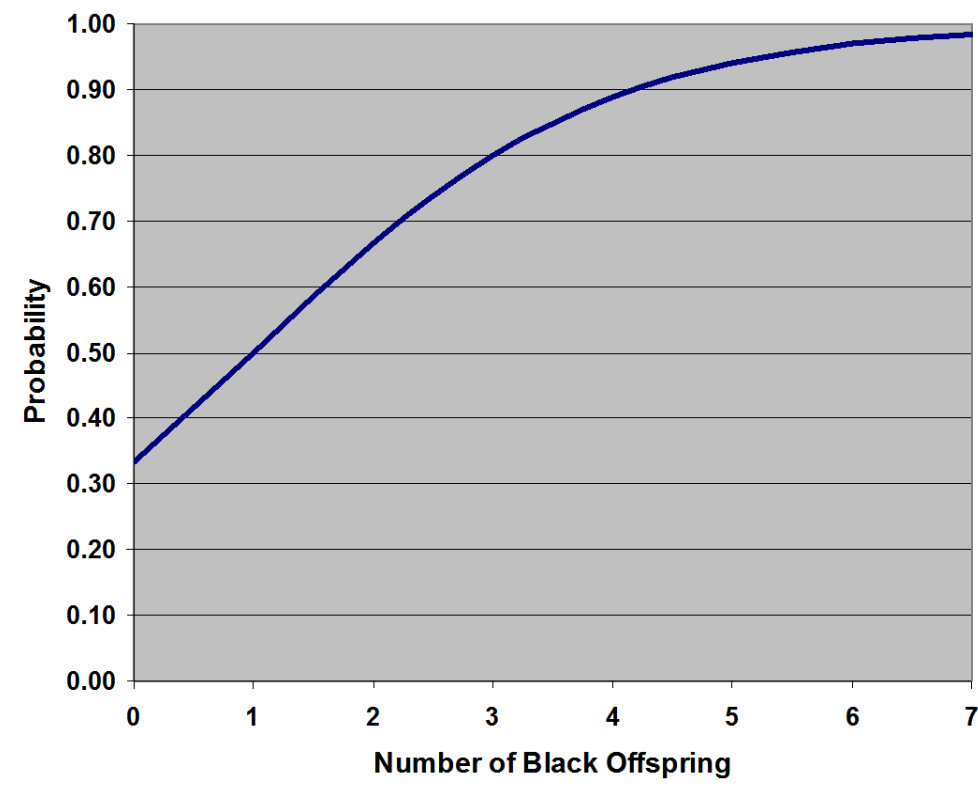

Figure 2. Probability that male parent mouse has two dominant color genes, given that a) the female parent has two recessive color genes, and b) a series of black offspring are born.

\section{Application of Bayesian Inference to Response Surface Modeling}

The examples in the previous section were offered as simple illustrations of Bayes' Theorem. We consider examples in this section that are more directly relevant to aerospace research. We begin with an example of how additional information can result in an important change in the structure of a response surface model. We will also give an example of how Bayesian inference can be used in the validation of computational models, and how it can be applied in circumstances in which repetitive experiments are executed on the same test article, as when an instrument such as a force balance is calibrated repeatedly over time.

\section{A. Model Building}

We introduced an example of a response surface model for lift coefficient as a function of the angles of attack and sideslip that had been fitted to experimental wind tunnel data over a limited range of the independent variables. An inference was made in the process of developing this model that the pure quadratic angle of attack term was not statistically significant at the 0.05 significance level. See Table 1 . However, the computed inference error associated with rejecting the null hypothesis for the regression coefficient of this term was still relatively small, albeit too large to meet our 0.05 criterion. We noted that results were available from another experiment on this same vehicle, which included a response surface modeling analysis similar to the one summarized in Table 1 for the first experiment.

In the analysis of the second data set, inference error probabilities associated with decisions to reject the null hypothesis were made for each term in the model, just as in the first experiment. As before, if the error probability exceeded 0.05 , the null hypothesis was not rejected, and the term was dropped from the model. If the error probability associated with rejecting the null hypothesis was sufficiently low $(<0.05)$, the term was retained in the model. For the second experiment, if we rejected the null hypothesis and therefore retained in the model the firstorder angle of attack, first-order angle of sideslip, and quadratic sideslip terms, the associated probabilities that these decisions would have been in error were again comfortably below our 0.05 criterion level, just as in the first experiment. Likewise, both experiments resulted in inference error probabilities well above the 0.05 criterion for rejecting the null hypothesis and retaining the interaction term in the full second-order model. So again we decided to retain both first order terms from Eq. (1), reject the interaction term, and retain the quadratic sideslip angle term. However, the two data sets resulted in different inferences with respect to the quadratic angle of attack term, as illustrated in Table 4.

Based on the first experiment we are unable to infer with at least $95 \%$ confidence that the regression coefficient for the quadratic angle of attack term is non-zero. We are therefore unable to report significant curvature in the lift coefficient as a function of angle of attack, and would conclude that we were not approaching the onset of stall in 
the angle of attack range examined in this experiment. We reach precisely the opposite conclusion from an analysis of the second experiment. A Bayesian analysis allows us to reconcile these results in such a way that proper weight is given to both experiments.

Table 4. Selected Characteristics of Quadratic Angle of Attack Term in Second-Order Response Surface Model of Lift Coefficient as a Function of the Angles of Attack and Sideslip.

\begin{tabular}{|r|c|c|}
\hline $\begin{array}{c}\text { Characteristics of Quadratic Angle } \\
\text { of Attack Term }\end{array}$ & First Experiment, Table 1 & Second Experiment \\
\hline Regression Coefficient & $-1.051 \mathrm{E}-03$ & $-1.190 \mathrm{E}-03$ \\
\hline Std Err in Coefficient & $5.48 \mathrm{E}-04$ & $4,90 \mathrm{E}-04$ \\
\hline Coefficient as Multiple of Std Err & 1.92 & 2.43 \\
\hline $\begin{array}{r}\text { Inference Error Probability if Term } \\
\text { Retained }\end{array}$ & $\mathbf{0 . 0 8 4 2}$ & $\mathbf{0 . 0 0 7 6}$ \\
\hline $\begin{array}{r}\text { Max acceptable Inference Error } \\
\text { Probability }\end{array}$ & 0.0500 & 0.0500 \\
\hline Inference & Reject Term (Err prob > 0.05) & Retain Term (Err prob <0.05) \\
\hline
\end{tabular}

Let us assume that our two estimates of the regression coefficient are random variables based on normally distributed data samples with means $\mu_{1}$ and $\mu_{2}$ and standard deviations $\sigma_{1}$ and $\sigma_{2}$, where the subscripts identify the two experiments. It is the convention to refer to one of these as the prior distribution and to say that it is revised by information from the other experiment. However, this does not imply any time-ordering. That is, the "prior" distribution does not have to have been established first. It is equally valid to say that conclusions based on results obtained in either experiment are revised because of information from the other one. However, we will arbitrarily declare the results displayed in Table 1 as having come from the prior distribution, which we will revise based on the additional results for the quadratic angle of attack term that are displayed in the right column of Table 4.

It can be shown ${ }^{9-11}$ that the posterior distribution in such a case is also normally distributed, with a mean, $\mu_{0}$, and a standard deviation, $\sigma_{0}$, represented as weighted combinations of the means and standard deviations of the two experimental data samples:

$$
\begin{gathered}
\mu_{0}=\frac{1}{w_{1}+w_{2}}\left(w_{1} \mu_{1}+w_{2} \mu_{2}\right) \\
\frac{1}{\sigma_{0}^{2}}=w_{1}+w_{2}
\end{gathered}
$$

Where the weighting functions, $w_{i}$, are

$$
w_{1}=\frac{1}{\sigma_{1}^{2}} \text { and } w_{2}=\frac{1}{\sigma_{2}^{2}}
$$

The mean of the posterior distribution for the regression coefficient is just a weighted average of the regression coefficient estimates from the two experiments, with the weighting determined by the uncertainty in each estimate. In this way, the greater weight is given to the estimate with the least uncertainty. The variance of the posterior distribution for the regression coefficient is based on a pooling of the variances from each experiment. 
After inserting numbers from Table 4 into Eqs. (16), we conclude that the posterior estimate of the regression coefficient for the quadratic angle of attack term in our lift coefficient model has a value of

$$
\mu_{0}=\left[\frac{1}{\frac{1}{\left(5.48 \times 10^{-4}\right)^{2}}+\frac{1}{\left(4.90 \times 10^{-4}\right)^{2}}}\right]\left[\frac{-1.051 \times 10^{-3}}{\left(5.48 \times 10^{-4}\right)^{2}}+\frac{-1.190 \times 10^{-3}}{\left(4.90 \times 10^{-4}\right)^{2}}\right]=-1.128 \times 10^{-3}
$$

with a standard deviation of

$$
\sigma_{0}=\sqrt{\frac{1}{\left(5.48 \times 10^{-4}\right)^{2}}+\frac{1}{\left(4.90 \times 10^{-4}\right)^{2}}}=3.653 \times 10^{-4}
$$

Figure 3 compares three normal distributions for the quadratic angle of attack regression coefficient. The black one is the prior distribution, the red one represents the new data, and the blue one is the posterior distribution, reflecting a Bayesian revision of the prior distribution per Eqs. (16) to reflect the information in the new data. (This figure displays positive means for the distributions consistency with Eq. (3), although the actual quadratic angle of attack regression coefficient is negative - concave down.)

By inserting values from Eqs. (17) into Eq. (3), the inference error probability associated with rejecting the null hypothesis for the posterior (revised) distribution is computed as 0.0010 , down from the 0.0842 value associated with the prior distribution. That is, the original odds against an inference error if we retained the quadratic angle of attack term were about 11 to 1 , or 1 chance in 12 of an erroneous inference. We had established our maximum acceptable odds at 19 to 1 , or 1 chance in 20 of an inference error. After taking into account the new data, if we infer that the quadratic angle of attack term should be retained in the response surface model, the odds against that inference being wrong increase dramatically, from 11:1 to 999:1, or only 1 chance in a thousand of an inference

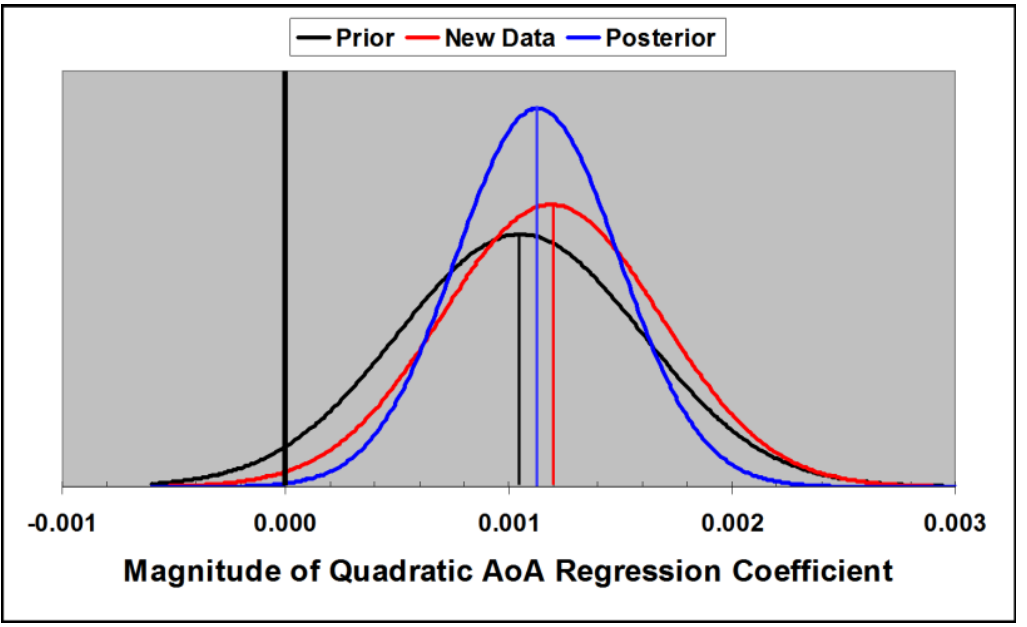

Figure 3. Probability distributions for quadratic angle of attack regression coefficient. Area under the curve to the left of zero represents the error probability for rejecting the null hypothesis. It exceeds 0.05 for the prior distribution (black) but not for the yellow (posterior) distribution, revised to account for new data (red). error, well within our risk tolerance. We are willing to assume that much risk in rejecting the null hypothesis for the quadratic angle of attack regression coefficient, and we therefore retain it in the model, concluding that the onset of stall is in fact evident over the range of angle of attack that we examined.

By incorporating additional information that allowed us to revise our prior conclusion about the significance of the quadratic angle of attack term, we achieved a substantial reduction in inference error risk. This was due in part to the fact that the signal to noise ratio for the second data set was greater than the first. The estimated regression coefficient was further from zero and the standard deviation in estimating the regression coefficient was less in the second data set than in the first.

The revised coefficient estimate is a weighted average of the coefficient estimated from each of the two data 
sets, and so it lies between the two individual estimates. However, it is still further from zero than in the prior experiment.

The prior variance was pooled with new data featuring less variance, which suggests that the revised variance would be smaller on that account. It is in fact a general result that pooling the variance associated with two samples results in less variance than either component sample. This can be seen clearly by combining Eqs. (16b) and (16c) as follows:

$$
\frac{1}{\sigma_{0}^{2}}=\frac{1}{\sigma_{1}^{2}}+\frac{1}{\sigma_{2}^{2}}=\frac{\sigma_{1}^{2}+\sigma_{2}^{2}}{\sigma_{1}^{2} \sigma_{2}^{2}} \rightarrow \sigma_{0}^{2}=\frac{\sigma_{1}^{2} \sigma_{2}^{2}}{\sigma_{1}^{2}+\sigma_{2}^{2}}
$$

It follows immediately that

$$
\sigma_{0}^{2}=\frac{\sigma_{1}^{2}}{1+\left(\sigma_{1} / \sigma_{2}\right)^{2}} \leq \sigma_{1}^{2}
$$

and

$$
\sigma_{0}^{2}=\frac{\sigma_{2}^{2}}{1+\left(\sigma_{2} / \sigma_{1}\right)^{2}} \leq \sigma_{2}^{2}
$$

The combination of a narrower probability distribution and a shift in the mean away from zero resulted in a greater signal to noise ratio and therefore a reduction in the probability of an improper inference associated with the conclusion that the regression coefficient was in fact non-zero. Figure 4 displays the regression coefficients for the prior and revised distributions as well as for the added data used to revise the prior. They are represented in this figure as multiples of the standard error in estimating them. There is a transparent colored box in Fig. 4 covering the range from minus two to plus two standard deviations. Coefficients lying within this box are too close to zero to be declared non-zero with at least $95 \%$ confidence, as our risk tolerance specification requires. Coefficients outside this box can be distinguished from zero with acceptable inference error risk.

Note that the original coefficient estimate was just inside the box, reflecting the $\sim 0.08$ inference error probability from Table 1 that just did exceed our 0.05 tolerance level. The regression coefficient for the new data is comfortably outside the $\pm 2 \sigma$ range and the revised regression coefficient, with a substantially smaller standard deviation, is a sufficient number of standard deviations away from zero that we incur relatively little risk by inferring that this coefficient is indeed real (non-zero) and therefore belongs in the model.

Even though the quadratic angle of attack term was the only one in doubt, we apply the Bayesian revision process captured in Eq. (16)

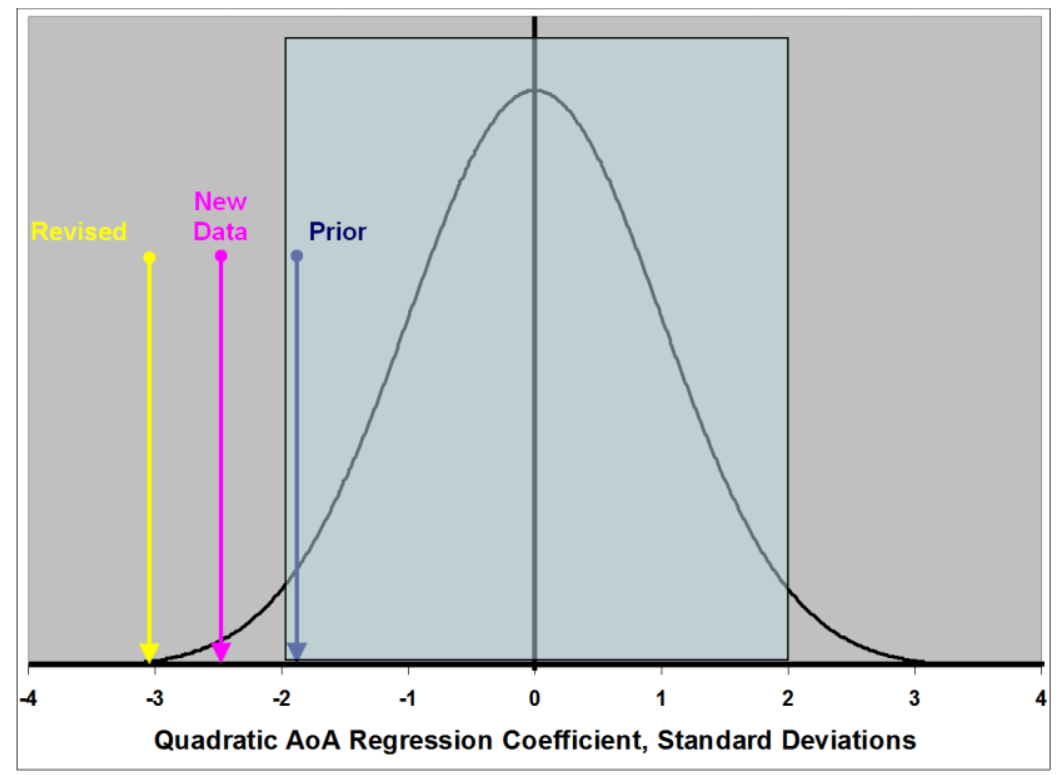

Figure 4. Quadratic angle of attack regression coefficient in multiples of standard deviation. Magnitude must be greater than 2 (positive or negative) to be resolvable from zero with at least $\mathbf{9 5 \%}$ confidence. Original (prior) estimate was just inside the limit. Estimate from new data was resolvable as was revised estimate. 
to each of the coefficients in the model to generate a revised model that reflects all the information in both data sets. We noted above that residuals from the model in Eq. (6), in which the quadratic angle of attack term is missing, had an average magnitude of 0.0013 , which exceeded our error budget of 0.0010 in this test by $30 \%$. We use the revised model in Eq. (20) to predict lift measurements for the same 16 combinations of angle of attack and angle of sideslip as before.

$$
y=b_{0}+b_{1} x_{1}+b_{2} x_{2}+b_{11} x_{1}^{2}+b_{22} x_{2}^{2}
$$

The revised model, Eq. (20), resulted in residuals with an average magnitude of 0.0008 , well within the error budget of 0.0010 , and reflecting a $38 \%$ reduction in prediction error relative to the initial model. Figure 5 compares the residuals of the prior and revised model with the error budget.

\section{B. Validation of Computational Models}

It is not uncommon for different computational codes to produce different results, notwithstanding the fact that they were developed to describe a common phenomenon. ${ }^{12}$ One might say that to at least some extent, this is the norm. $^{13}$ When multiple computational codes are evaluated, their predictions might be compared with some reference to determine how great a difference there is between the prediction of each code and that reference. The reference could be a measurement of the physical phenomenon which the codes seek to predict, or absent any suitable physical measurement to serve as a standard, it might simply be the mean of all code predictions.

Consider a case in which, for simplicity, we assume that there are only two different computational codes, and that the validity of each is to be assessed by comparing predictions with a physical measurement. There will obviously be uncertainty in the physical measurement used as a reference, in the form of ordinary experimental error. Each code will also have uncertainty, notwithstanding the absence of variance in replicated computations. ${ }^{\dagger}$ As a general rule, the degree of uncertainty in a code prediction depends on the combination of independent variable levels for which the prediction is made. There are several reasons for this, including the fact that the slope of the response function will generally vary over the design space, resulting in a greater or lesser impact of uncertainties in the independent variable settings.

For the purpose of this example, we assume that each code is intended to quantify drag on a specified test aircraft, and that its ability to do so will be evaluated by comparing code predictions with flight data acquired at a certain set of conditions. We assume further that the uncertainty in flight measurements has been quantified through an appropriate analysis of test data, and that the uncertainty associated with predictions made by each computational code has been estimated.

Let us say that flight conditions are chosen for this comparison such that the empirically determined drag is 700 counts with a standard deviation of 30 counts. The first code estimates the drag to be 750 counts for these same conditions, with a standard error of 10 counts. The second code estimates the drag for these conditions to be 650 counts, but it is only capable of rather less precise estimates, characterized by a standard error of 60 counts.

At first glance it might seem as if both codes performed comparably in that they each produced predictions that differed from the measured flight data by identical amounts -50 counts. However, the simple

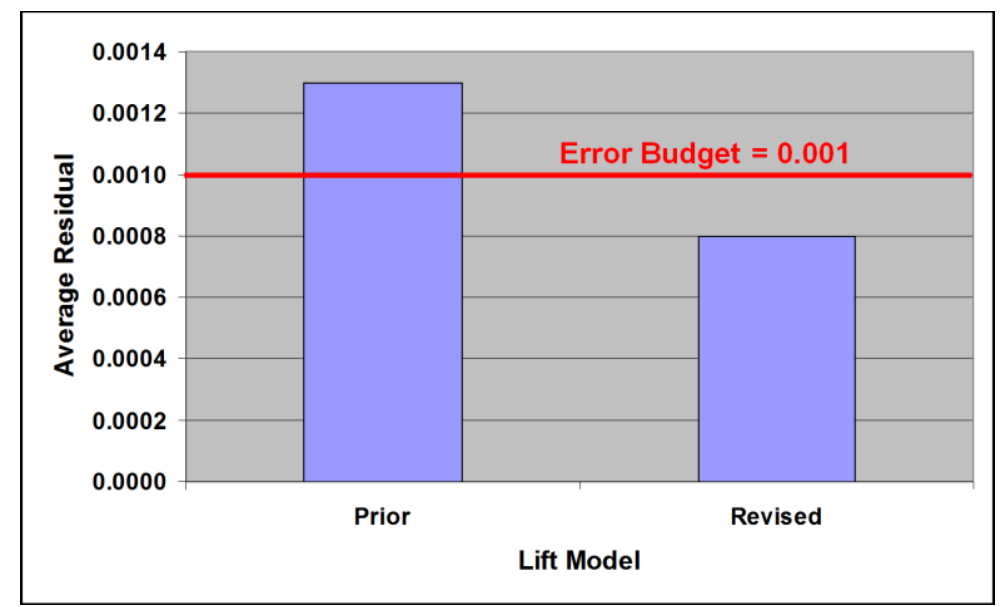

Figure 5. Residuals from the prior response surface model exceeded the error budget. Bayesian inference justified the addition of an additional term to the model that resulted in residuals that were within tolerance.

\footnotetext{
$\dagger$ The fact that a computational code will produce the same numerical result without variance for any number of replicates does not suggest that there is no uncertainty in a given code prediction. It simply implies that the uncertainty in a computational code cannot be quantified by replication.
} 
comparison is potentially misleading for two reasons. First, both of the computational estimates as well as the measured drag are random variables, describable in terms of probability distributions that are characterized by dispersion metrics as well as location metrics. Comparing each code prediction with the experimental data only on the basis of their location metrics (means of their probability distributions) fails to account for the dispersion (variance) in each estimate. Furthermore, the measured drag is itself no more than an estimate, subject to uncertainty just as the computational estimates are. The assumption that the measured estimate should be given more weight than the computational estimates is only valid if the uncertainty in the measured estimate is significantly less than the uncertainty in either computed value. That is not true in this case, and in fact the standard deviation for Code 1 is even smaller than the standard deviation for this particular sample of flight data.

In using the measured data to assess the computational results, we should ask how much the sponsor of each code is able to learn by an exposure to the measured results. We can use Bayesian revision to address this question. We have the mean and standard deviation of each code's distribution prior to the data, and the mean and standard deviation of the data. We can therefore use Eqs. (16) to compute the posterior distributions when each code's prior distribution is revised by the information in the data. Means and standard deviations for the prior and posterior code distributions are listed in Table 5. Figure 6 compares these distributions.
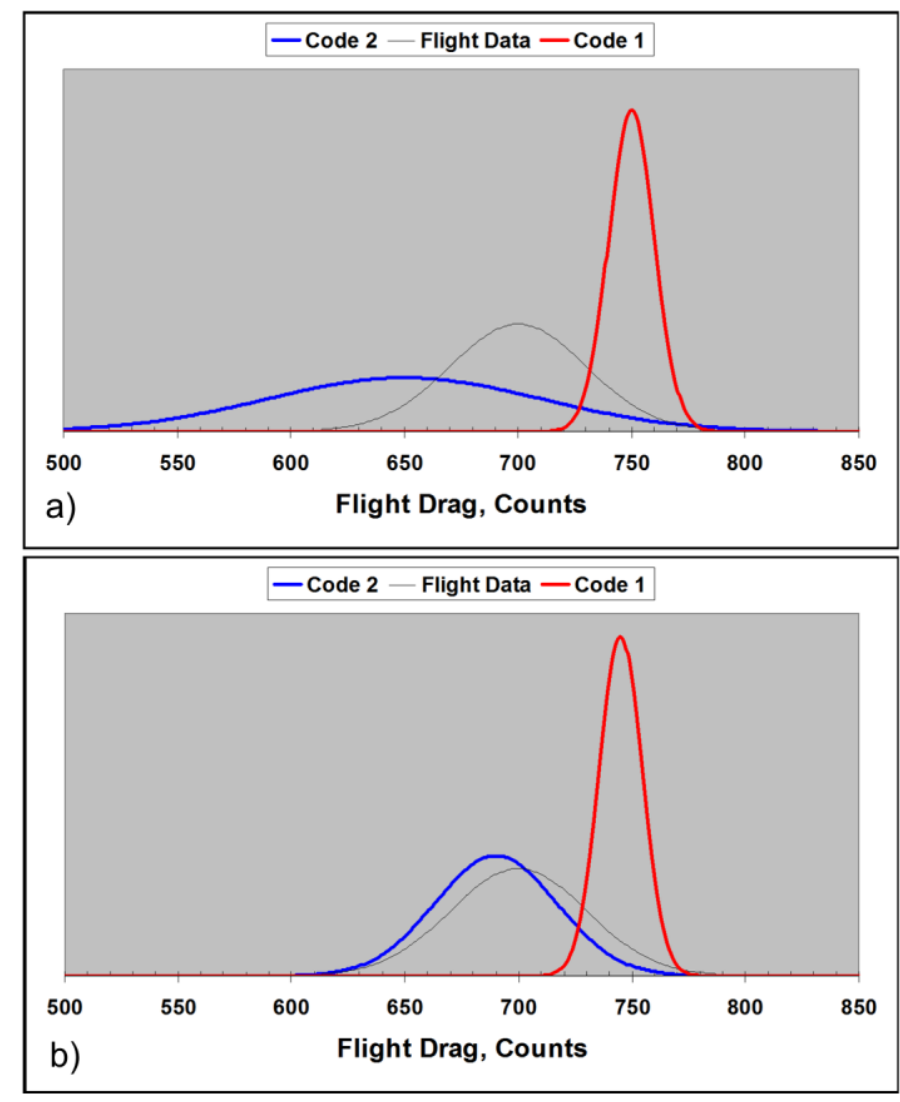

Figure 6. Flight drag distributions for computational codes 1 and 2 (red and blue, respectively), compared to the distribution for measured flight drag (black). a) Distributions prior to revision by data, b) Distributions after revision by data.

Table 5. Mean and standard deviation for prior and revised distributions for two computational codes compared with physical measurement.

\begin{tabular}{|r|c|c|c|c|c|}
\hline \multirow{2}{*}{ Measurement } & \multicolumn{2}{|c|}{ Code 1 } & \multicolumn{2}{c|}{ Code 2 } \\
\cline { 3 - 6 } & & Prior & Revised & Prior & Revised \\
\hline Mean & 700 & 750 & 745 & 650 & 690 \\
\hline Standard Deviation & 30 & 10 & 9.5 & 60 & 26.8 \\
\hline
\end{tabular}

Table 5 and Fig. 6 each reveal a similar story. The revised means for Code 1 and Code 2 are much closer than the prior means; the prior means differed by 100 drag counts while the posterior or revised means differ by only 55 counts. Likewise, the standard deviations differed by 50 counts originally, but only by 17.3 counts after revision.

Clearly the sponsors of Code 2 learned more by their exposure to the measured data than the sponsors of Code 1 in this example. The sponsors of Code 1 only needed to revise their drag estimate from 750 to 745 after seeing the data, a scant five-count change of opinion. The Code 2 sponsors changed their estimate from 650 counts to 690 , a 40-count change. Likewise, the measured drag data only caused the Code 1 sponsors to tighten their standard deviation estimate from 10 to 9.5, a mere half-count revision, while the Code 2 sponsors changed their standard deviation estimate from 60 counts to 26.8 after seeing the measured drag data, a change of 33.2 counts. 
Note how differently we regard the two computational codes after this type of comparative analysis. Originally we were willing to conclude that little distinguished one code from the other, in that they each predicted the measured drag with the same error. After accounting for the relative uncertainty in each code prediction (and in the confirming measurement!), we see that conclusions based on one of the codes would be altered substantially more by the experimental observations than the other.

The reason is that the uncertainty in drag estimates made by one of the codes was substantially greater than the other code; users of one code would have only a relatively vague idea of the drag they were estimating, while users of the other code would have a rather more precise estimate. In fact, in this example the users of Code 2 had less uncertainty in their estimates than the experimentalists providing the confirmatory measurement, while explains why their posterior knowledge was revised so little as a result of their exposure to this additional information. On the other hand, users of Code 1 began with a relatively imprecise prediction of the measured drag, and their estimates were subject to rather greater revision as a result of the measurement.

We can see this situation graphically in Fig. 6a, where the prior distribution for Code 2 is considerably more peaked than the prior distribution for Code 1, and it is even rather more peaked than the distribution for the physical measurement. For this reason the data has rather little influence on the revised distribution of Code 1, while it has a fairly dramatic influence on the revised distribution of Code 2, as seen in Fig. 6 b.

We noted above an alternative for evaluating individual computational codes when there is no physical measurement available to serve as a reference against which to make comparisons. It is a customary assumption that when multiple estimates of some phenomenon are available, the median or the mean of those estimates is a more reliable estimator than any one individual estimate. It is likewise customary to regard estimates that are the farthest from this reference to be the least reliable ("outliers," if they are sufficiently far away). This suggests that some appropriately weighted combination of computational code predictions might serve as a suitable reference against which to measure individual predictions. At the very least, the variance in an ensemble of computational code estimates can be regarded as an indicator of the state of the art for computational predictions. ${ }^{14}$

Bayesian methods summarized in this paper are well suited for generating a weighted reference prediction against which to compare individual codes. Eqs. (16) suggest that a rational weighting could be based on the uncertainty associated with each individual code, with those codes featuring the least uncertainty weighted the most and those featuring the greatest uncertainty weighted the least.

It is beyond the scope of this paper to consider explicit methods for estimating the uncertainty of individual computational codes, except to say that this is an area of increasing interest in the computational aerospace community. The author has offered some ideas at what at this writing has been the most recent conference devoted exclusively to this topic, ${ }^{15}$ and the literature of computational uncertainty continues to grow. For purposes of illustration, we assume that some rational means exists by which to assign uncertainty to the response predictions made by each computational code. Also for illustration, we assume that three computational codes have produced estimates for flight drag for identical flight conditions. We will use for illustration the same estimates reported in Table 5 for Codes 1 and 2, and assume now that the physical measurement reported in that table describes the prior distribution for a third computational code. Equations 16 extend naturally to multiple distributions. For prior means of 650,700 , and 750 , with corresponding standard deviations of 60,30 , and 10 , these equations result in a mean weighted by the reciprocal of variance in each estimate of 742.7, with a pooled standard deviation of 9.4. These calculations are summarized in Table 6 .

Table 6. Comparison of three codes with and without accounting for uncertainty in predictions.

\begin{tabular}{|c|c|c|c|c|}
\hline & & Code 1 & Code 2 & Code 3 \\
\hline & Mean & 750 & 650 & 700 \\
\hline Standz & Deviation, $\sigma$ & 10 & 60 & 30 \\
\hline Docidnel & $\begin{array}{r}\text { Weighting } \\
\text { by } 1 / \sigma^{2}\end{array}$ & $750-742.7=7.3$ & $650-742.7=-92.7$ & $700-742.7=-42.7$ \\
\hline Resiaual & $\begin{array}{r}\text { No } \\
\text { Weighting }\end{array}$ & $750-700=50$ & $650-700=-50$ & $700-700=0$ \\
\hline Rankino & $\begin{array}{r}\text { Weighting } \\
\text { by } 1 / \sigma^{2} \\
\end{array}$ & 1 & 3 & 2 \\
\hline Kanking & $\begin{array}{r}\text { No } \\
\text { Weighting }\end{array}$ & 2 & 2 & 1 \\
\hline
\end{tabular}


Note how differently the code predictions are ranked if the reference is computed by weighting each prediction by the information it contains, as quantified by the reciprocal of the variance in each estimate. With no weighting, Code 3 has the highest ranking by virtue of the fact that its prediction lies squarely in the center of this three-point sample. Codes 1 and 2 tie for second place and are regarded as indistinguishable since their predicted responses are equally displaced from the sample median.

However, when a weighted mean is constructed that accounts for the uncertainty in each prediction, an entirely different picture emerges. Now Code 1 is regarded as having made the most reliable prediction, based on its relatively small variance (or equivalently, the relatively large amount of information contained in its estimate). Code 2 , regarded as equivalent to Code 1 when prediction uncertainty was ignored, is now ranked in third place, again because its relatively large variance translates into only rather vague predictions of flight drag for the combination of independent variables considered in this comparison. Code 3, ranked \#1 when prediction uncertainty was ignored, is now in second place behind Code 1 because its variance is three times larger than that of Code 1.

Obviously the utility of a Bayesian approach to computational code evaluation depends critically on the quality of uncertainty estimates in the individual code predictions. This argues for continued attention to the problem of assessing computational uncertainty.

\section{Instrumentation Calibration}

We consider as our last example of the application of Bayesian inference to response surface methods a special case in which an experiment is replicated on the same test article. This situation arises from time to time in different contexts, but it is the norm for experiments designed to calibrate measurement systems or individual instruments. The same experiment is typically executed each time an instrument is calibrated. To serve as a specific and common example, we will consider a force balance that is calibrated repeatedly over time.

It is customary in a force balance calibration experiment to consider only the latest calibration results in defining the coefficients of the transfer function that relates the balance's electrical output to the applied forces and moments. Notwithstanding the fact that this same balance may have been calibrated on many previous occasions, the results of those prior calibrations are seldom taken into account except for occasional comparisons to determine the general level of agreement with the most recent calibration. Since earlier calibration results will have been obtained with considerable expense and effort, it seems wasteful to simply discard all of that prior information each time the instrument is calibrated anew.

Bayesian inference models human learning by providing a mechanism for revising prior knowledge to reflect new information. In the context of a calibration experiment, this implies that information obtained in prior calibrations need not be lost each time an instrument is recalibrated. The calibration experiment can be regarded as an opportunity to obtain additional information that can be used to revise prior calibration results.

Force balances are calibrated by applying a prescribed schedule of loads and recording the corresponding electrical outputs. These data are typically fitted to a calibration model using linear regression. This model can be used to predict electrical outputs as a function of loads, and is typically inverted to enable the specification of applied loads for a given combination of electrical outputs recorded in a wind tunnel test, say.

There are different force balance designs in use but a common balance configuration measures three components of force (normal, axial, and side force with respect to a specified coordinate system), and three moment components (pitching moment about the side force axis, rolling moment about the axial force axis, and yawing moment about the normal force axis). Force balances are designed with the goal of producing a linear instrument capable of independent force and moment measurements. If that idealized goal could be achieved, then a simple first-order polynomial in six independent variables would be adequate to describe the relationship between any combination of forces and moments experienced by the balance and the electrical output produced for each force and moment component.

$$
y=b_{0}+b_{1} x_{1}+b_{2} x_{2}+b_{3} x_{3}+b_{4} x_{4}+b_{5} x_{5}+b_{6} x_{6}
$$

where $y$ is the electrical output for any one of the six forces and moments (often some combination of strain gage outputs), the $b_{i}$ are regression coefficients, and the $x_{i}$ are applied loads.

Unfortunately, a perfectly linear force balance with totally independent measures of the forces and moments is a mathematical abstraction that has proven elusive to engineer in practice. Typically there are some small interactions across the channels of a real force balance - the sensitivity or change in electrical output as one force or moment changes typically depends on the levels of other forces and moments that are simultaneously applied, for example. In addition to such interactions, there is generally some pure non-linearity (interactions of loads with themselves). 
For example, the change in electrical output corresponding to a unit change in applied load if often different for low loads than high loads. The electrical output is in such cases is a second-order (or higher) function of applied pure loads. The end result of this interactive and non-linear behavior is that a rather more complex calibration model is needed than the one described in Eq. (21).

For a single-piece six-component force balance, a second-order response model in six independent variables has been traditionally fitted in the calibration process, requiring 15 interaction terms and six pure quadratic terms in addition to the seven terms in Eq. (21), for a total of 28 terms in the model. Recent studies have indicated that thirdorder terms may improve the balance calibrations, which imply up to 84 mathematically possible candidate terms in the calibration equation for a six-component single-piece balance. In actual practice, most of these terms are either non-existent (terms that correspond to an interaction that does not occur naturally in a given balance, for example), or so small that the additional uncertainty they contribute to the calibration model exceeds their influence on model predictions. In either case, retaining such terms would reduce the overall signal to noise ratio of the calibration.

Ulbrich and Volden at NASA Ames Research Center have pioneered the development of new methods and software tools to identify and eliminate negligible terms from potentially large balance calibration models, resulting in compact math models and improved balance calibrations. ${ }^{16-20}$ Parker, et. al. ${ }^{21-25}$ DeLoach and Philipsen, ${ }^{26}$ and Philipsen and Zhai ${ }^{27}$ have examined the use of specialized balance calibration hardware and the design of compact balance calibration experiments facilitated by such hardware that are scaled for reduced calibration models.

A balance calibration experiment has in common with all response surface modeling experiments a process in which decisions are made to reject or retain candidate terms in a proposed model. This process was illustrated with the first example in this section of the paper, in which null hypotheses are established for each candidate term, and an inference is made as to whether that hypothesis should or should not be rejected. Each inference has a non-zero probability of being in error, which can be estimated and which is compared with a prescribed maximum acceptable inference error probability. Terms are rejected or retained in the model based on how the associated inference error probability compares with what is deemed acceptable. A consensus is reached and documented with respect to acceptable levels of inference error risk early in the formal experiment design process.

It is possible to apply Bayesian inference methods outlined in this paper by using current calibration results to revise prior inference error probabilities associated with each term in the calibration model. In this way we can modify what we already know about the model with information recently obtained. The pooling of variances that occurs with each new calibration results in progressively higher precision inferences with respect to decisions to retain or reject terms from the model. Note that retention decisions are generally unambiguous (vanishingly small inference error probabilities) for the first-order terms in a balance calibration model, and can also be quite clear for the relatively strong interaction terms. However, for numerous model terms with smaller regression coefficients, the decision to retain or reject that term can turn on the level of unexplained variance in the calibration data or other subtle effects. It is possible that such terms might be retained in one calibration and rejected in another.

This circumstance gives rise to an interesting model selection opportunity. Imagine a series of calibrations that have been performed on the same balance. The first-order terms and larger interaction terms will have been retained in the response model for every calibration with negligible inference error probability, however some of the terms with smaller regression coefficients may be retained in some calibrations and rejected in others. That is, as a result of the most recent calibration data, one might decide to include a term that was previously rejected or reject a term that was previously retained in the model. This gives rise to the notion of transitions from one calibration to another between one state in which a given term is retained in the model, and another state in which it is rejected.

Over a sufficient number of calibrations, one could begin to estimate conditional transition probabilities. If we let the subscripts " 0 " and " 1 " refer to states in which a given term is missing or present in the model, respectively, we can define $p_{00}$ as the probability that a term that was rejected from the model in the last calibration would be also rejected in the current calibration, and $p_{01}$ would represent the probability that a term rejected the last time would be retained this time. (Obviously, these two probabilities sum to 1.) Likewise, $p_{10}$ represents the probability that a term retained in the prior calibration would be rejected in the current one, and $p_{11}$ represents the probability that a given term is retained in two consecutive calibrations. These latter two probabilities also sum to 1 .

Let us now define $\pi_{0}$ and $\pi_{1}$ as elements of a $1 \times 2$ column vector, $\Pi$, describing the proportion of times that a given term has been rejected or retained. We assign the four transition probabilities to elements of a transition probability matrix, $\mathbf{P}$, as follows:

$$
\mathbf{P}=\left[\begin{array}{ll}
p_{00} & p_{10} \\
p_{01} & p_{11}
\end{array}\right]
$$


We then have $=\mathbf{P} \Pi_{i}=\Pi_{\mathbf{i}+\mathbf{1}}$ which expands as follows:

$$
\begin{aligned}
& p_{00} \pi_{0, i}+p_{10} \pi_{1, i}=\pi_{0, i+1} \\
& p_{01} \pi_{0, i}+p_{11} \pi_{1, i}=\pi_{1, i+1}
\end{aligned}
$$

Equation (23a) simply states that the fraction of the total number of calibrations in which a given term was inferred to be in state " 0 " (rejected) is, after the $(\mathrm{i}+1)^{\text {st }}$ calibration, equals the fraction that were in that state after the $\mathrm{i}^{\text {th }}$ calibration and stayed in that state, plus the fraction that were in state " 1 " (retained) after the $\mathrm{i}^{\text {th }}$ calibration that were then rejected in the $(i+1)^{\text {st }}$ calibration. Equation (23b) similarly describes the proportion in state " 1 " (retained) after the $(i+1)^{\text {st }}$ calibration as the sum of those starting in state " 0 " and transitioning to state " 1 " plus those starting in state " 1 " and staying there.

We have described a series of discreet events (calibrations) in which the state of a given term in the calibration model after each calibration (retained or rejected) is a random variable (i.e., not deterministic but defined by some probability). This chain of events has what is known as the "Markov property," in that given the state transition probability matrix, Eq. (22), the state of each term in the calibration model depends only on the state of that term after the last calibration. Such a process is known mathematically as a Markov chain.

Markov chains have certain properties that we can exploit in this analysis. Specifically, given Eqs. (23), the vector $\Pi$ is an equilibrium distribution if its elements $\pi_{j}$ sum to 1 . That is certainly the case for this Markov chain; the probability that a given term in the model will either be retained or rejected after each calibration is always 1 . The fact that the vector $\Pi$ is therefore an equilibrium distribution means that the percentage of times a given term will be rejected (or retained) approaches a constant after a sufficient number of calibrations. That constant depends only on the transition probability matrix, $\mathbf{P}$, so the system converges to a final equilibrium state distribution that is completely independent of the starting distribution.

We can compute the equilibrium state distribution for each term (i.e., the long-term probability that the term will be retained or rejected) by noting that at equilibrium, $\Pi_{i}=\Pi_{i+1}$, so that Eqs. (23) become:

$$
\begin{aligned}
& p_{00} \pi_{0}+p_{10} \pi_{1}=\pi_{0} \\
& p_{01} \pi_{0}+p_{11} \pi_{1}=\pi_{1}
\end{aligned}
$$

Combining this with the fact that $\pi_{0}+\pi_{1}=1$, yields the following formulas for the equilibrium state distribution probabilities $\pi_{0}$ and $\pi_{1:}$

$$
\begin{aligned}
& \pi_{0}=\frac{p_{10}}{p_{01}+p_{10}} \\
& \pi_{1}=\frac{p_{01}}{p_{01}+p_{10}}
\end{aligned}
$$

We can examine certain special cases. If $p_{10}=0$ (no instances in which a term that appears in the model is ever rejected in a subsequent calibration), then $\pi_{0}=0, \pi_{1}=1$, and the probability is $100 \%$ that this term will be in the calibration model. If $p_{01}=0$ (no instances in which a rejected term ever appears in the model in a subsequent calibration), then $\pi_{0}=1$ and $\pi_{1}=0$, so the long-term probability that this term will ever appear in the calibration model is zero. If $p_{10}$ and $p_{01}$ are such that $\pi_{0}$ represents an acceptably low probability of an inference error for a 
given term, then the decision to retain that term can be made for all subsequent calibrations of this balance. If $p^{\prime}$ represents that critical probability, we have the following condition for $\pi_{0}$ :

$$
\pi_{0}=\frac{p_{10}}{p_{01}+p_{10}} \leq p^{\prime} \rightarrow p_{10} \leq p^{\prime}\left(p_{01}+p_{10}\right)
$$

or

$$
\pi_{0} \leq p^{\prime} \rightarrow p_{01} \geq\left(\frac{1-p^{\prime}}{p^{\prime}}\right) p_{10}
$$

Equation (27) informs us that if, for example, $p^{\prime}=0.05$ as is a common convention, then we must have $p_{01} \geq 19 p_{10}$ as a condition for retaining the term. That is, we incur less than a $5 \%$ probability of an inference error if we retain a term for which the long-term 0-to-1 state transition probability is at least 19 times greater than the longterm 1-to-0 state transition probability. Such probabilities are revealed through a series of calibrations, with prior calibrations contributing equally to the model selection process.

In general, we expect that consistent calibration methods will result in relatively large or relatively small values of $\mathrm{p}_{01}$ and $p_{10}$. That is, we do not expect terms to be equally likely to be retained in one calibration and rejected in another if the calibration process is consistent. This is only likely to occur when the magnitude of the regression coefficient is on the order of the uncertainty in estimating it, so that slight variations in the unexplained variance from calibration to calibration are sufficient to either mask the term or to marginally reveal it. In such circumstances we would tend to discard the term on the grounds that such small regression coefficients are not likely to make a practical difference in response model predictions, while each retained term carries with it some incremental contribution to the total prediction variance, per Eq. (2). That is, when in doubt we yield to a prejudice in favor of rejecting terms rather than retaining them.

\section{Discussion}

Bayesian inference is especially relevant to response surface modeling experiments such as wind tunnel tests because of the critical role that reference distributions and formal hypothesis testing play in the construction of response surface models. We represent an unknown response function as a Taylor series which for mild and commonly occurring conditions is an exact representation provided we retain an infinite number of terms. Resource constraints prevent the acquisition of sufficient data to fit an infinite number of terms, and in any event such extravagance is not necessary in practical circumstances. We are, however, always faced with the problem of inferring which terms to reject and which to retain. We make objective retention/rejection decisions by noting the location of a regression coefficient estimate relative to a reference distribution with a mean of zero and a standard deviation reflecting the uncertainty in estimating the coefficient. If the coefficient estimate is located a sufficient number of standard deviations away from zero, we retain that term in the model. Otherwise, we reject it.

Bayesian inference facilitates "learning by experience" in that it permits prior knowledge to be revised by additional information. The role that such prior information plays has been a source of controversy historically, with critics of the Bayesian approach noting that reliance upon prior information in some sense diminishes what the current data "is telling us." Bayes himself recognized this potential criticism, and suggested in what came to be known as Bayes' Postulate that such objections could be overcome by assuming a uniform prior distribution in circumstances for which little is known a priori. He apparently was sufficiently unsure of this point that he did not publish it, however. Bayes' views only came to light when his work was published after his death by his friend, Richard Price ${ }^{28}$.

Advocates of Bayes' methods note that his basic theorem (Eq. (9), restated as Eq. (13)) is a straightforward statement of conditional probability and is not in and of itself in dispute. It is rendered controversial by considerations of the role that the prior distribution plays as noted above, and also by fundamental notions of the meaning of probability. The conventional (or "frequentist") view of probability is that it is related to the frequency with which events can be expected to occur. Bayesians adopt a more liberal interpretation, associating probability with the extent to which a given viewpoint is held. A Bayesian would assign a high probability of occurrence to an event he felt was likely to occur, for example. This leads to the notion of subjective prior distributions, a significant point of contention among frequentists who argue that posterior means can be influenced to take on virtually any 
value by a suitably selected subjective prior. This renders statistical inference, they claim, much too vulnerable to apriori prejudices. On the other hand, Bayesians point to circumstances in which suitable frequency-based probability information is unavailable and yet inferences must be made, in which case good-faith subjective probability estimates based on experience have a role to play. Examples include inferences with respect to rare disasters such as aircraft accidents or nuclear power plant failures, or other decision-making circumstances in which limited prior information is available, such as whether to drill for oil at a given location. In such cases, an informed but nonetheless subjective evaluation may constitute much of what is believed to be true before the introduction of what might in fact be rather meager additional evidence.

Box and Tiao $^{29}$ enumerate and systematically address issues surrounding the prior distribution, and develop a practical variation of Bayes' Postulate involving locally uniform prior distributions. It was noted above in the discussion of Fig. 6 that the relative influence of the prior distribution and the additional data used to revise it depends on the "peakiness" of the probability distributions. For circumstances in which the precision of the new information is significantly greater than that of the prior information, the new information will dominate the prior in defining the posterior distribution. For practical circumstances in scientific inquiry this is generally the case, as investigations are seldom undertaken absent the prospects of some resulting increase in knowledge, which implies a dominant role for the new information and a less influential role for the prior distribution. (In short, if we "knew" the answer a priori, we would not have undertaken the experiment.) For the response surface modeling applications considered in this paper, there is even less of a controversy. The prior distributions considered in such applications involve an interpretation of probability that is generally consistent with conventional frequentist notions.

Bayesian inference is appealing for scientific research because it is based on a distinction between what is true in nature, and what is inferred. The cruise lift of a new aircraft may or may not in fact exceed some minimum certification requirement. But in either case we might have to make an inference about whether it does or does not based on observations we have made of a model airplane set to emulate cruise conditions in a wind tunnel. It is possible for the results of a wind tunnel test to suggest that the lift is adequate when it is in fact adequate, but it is also possible that the results will suggest the lift is adequate when it is not. Bayesian inference provides a mechanism for explicitly recognizing such limitations of experimentation. When we use Bayesian inference to evaluate the probability that " $\mathrm{A}$ " is true given that we have observed "B," we not only consider the probability of observing " $B$ " when " $A$ " is true, but also the probability of observing " $B$ " when "A" is not true. See Eq. (13). That is, we are forced to take into account the fact that our measurements are imperfect, and that it is possible to obtain an indication that something is true both when it is true and when it is not true.

To illustrate this important point, consider an example drawn from the news just prior to the conference in which this paper is presented. In December of 2007, a report was presented to the commissioner of major league baseball, Mr. Bud Selig, on the use of steroids among professional baseball players. The report was accompanied by significant publicity, and amounted to a severe indictment of the sport and many of its key players.

The author downloaded the full report and performed keyword searches on such seemingly relevant words as "accuracy" and "precision" to gain some insight into the reliability of the tests used to detect steroids. The word "accuracy" appears exactly once in the 311-page report (in a commentary about an eyewitness report) and the word "precision" does not appear at all. Similar searches for such strings as "inference," "false positives," and "false negatives" returned no hits. On the other hand, a Web search revealed a site apparently devoted to the topic of steroids in baseball that listed the names of 12 players who had experienced the league's 10-day suspension rule for first-time offenses, a number nominally consistent with the Commissioner's assertion that $1.2 \%$ of the players had used steroids last season, based on a combined American League and National League roster of 854 players.

We will augment this meager information with what are believed to be reasonable suppositions to illustrate how a Bayesian analysis might be performed on the steroids-in-baseball scandal. (The central points of this illustration are not dependent on the suppositions.)

Let us assume that a steroid test is "95\% accurate," by which it is meant that if a player who uses steroids is subjected to this test, he will test positive $95 \%$ of the time. This means that $5 \%$ of steroid users will pass this test. Let us also assume that the test will exonerate non-users $95 \%$ of the time, which nonetheless means that $5 \%$ of those who do not use steroids will be falsely accused.

If we use for this illustration the figure cited above by the Commissioner of $1.2 \%$ as representative of the fraction of players who actually do use steroids, we can easily compute how many users and non-users will test positive. We assume that 0.012 of the league uses steroids and 0.95 of those will test positive, so $0.012 \times 0.95=$ 0.0114 of the league will be users who test positive. On the other hand, $1-0.012=0.988$ is the fraction of the league that is clean in this example, of which 0.05 will wrongly test positive. So $0.988 \times 0.05=0.0494$ is the fraction of the league that will be falsely accused. We will have $0.0114+0.0494=0.0608$ as the total fraction of the league that will test positive (roughly 52 players), of which only $0.0114 \times 854$, or about 10 , will actually be steroid 
users. Therefore, the probability that a player actually does use steroids, given that he has tested positive, is only about 10 in 54, or roughly $18.5 \%$.

The probabilities of false negatives and false positives were estimated in this example for the sake of illustration, although they are believed to be not unrealistic. The total number of positive tests computed in this example-52is consistent with numbers reported in the media for active players said to have tested positive for steroid use.

The calculations presented in this example were rounded to ensure an integer number in each category of accused players (correctly accused and falsely accused). An exact calculation would invoke Eq. (13), with "A" corresponding to a player actually being a steroid user and " $\mathrm{B}$ " corresponding to a positive test. In this example we would have

$$
\begin{aligned}
& P(A)=0.012 \\
& P(B \mid A)=0.95 \\
& P(B \mid \bar{A})=0.05 \\
& P(\bar{A})=1-0.012=0.988 \\
& \therefore P(A \mid B)=\frac{P(B \mid A) P(A)}{P(B \mid A) P(A)+P(B \mid \bar{A}) P(\bar{A})}=\frac{0.95 \times 0.012}{(0.95 \times 0.012)+(0.05 \times 0.988)}=0.1875
\end{aligned}
$$

as the exact solution, which compares with our $18.5 \%$ approximation.

These results illustrate how much difference there can be between what is true in nature, and what is inferred in a test. There is only an $18.75 \%$ probability that a player who tests positive with this test will actually be a steroid user, notwithstanding the " $95 \%$ reliability" of the test. This is an example of how inferences can be quite different when measurement imperfections are explicitly taken into account, as they are when Bayesian inference is applied.

\section{Concluding Remarks}

This paper has provided an elementary introduction to the subject of Bayesian inference as it might be applied to experimental aerospace research in general, and experimental aeronautics in particular. Examples have been provided to show the applicability of the method to response surface modeling, to the validation of computational models, and to tasks which involve repeated experiments on the same test article, as in the calibration of an instrument over time.

Bayesian inference extends insights available from a conventional frequentist perspective of statistics by explicitly accounting for the inevitable limitations of any system or process used to observe nature. It assumes we originally hold certain beliefs with some uncertainty, and that we try to perfect our knowledge by the introduction of new information that is also imperfect. This method encompasses as a special case the common situation in experimental research when we hold no particular beliefs a-priori; that is, when the probability associated with a given inference is 0.5 before we have acquired any new information in the form of experimental data.

Bayesian inference has special practical utility in that it models the learning process that is fundamental to scientific research, and that is an explicit element of the Modern Design of Experiments. We make progress in MDOE through a series of cycles in which we alternately play the role of advocate and critic of a given proposition. We advance a proposed model to describe nature in certain limiting circumstances, and then subject that model to criticism that is often based upon the introduction of new information, itself imperfect. Throughout this process we modify our original perceptions to account for the new information, rather than discarding them wholesale in preference for a completely new set of suppositions based on the latest observations. We then subject our revised models to criticism, and so on, until we are satisfied that they are adequate for a particular purpose. This provides a mechanism of handing down information, often hard-won at great expense, from preceding cycles of investigation through to future investigators.

The Modern Design of Experiments is based upon an intrinsically statistical framework in which a series of inferences are made about the suitability of incorporating progressively higher-order terms in a series representation of some system response, modeled as a function of specified independent variables. We make inferences for each term in the proposed model by testing a null hypothesis that asserts a-priori that the term under evaluation is 
insignificant and does not belong in the model. We only retain such a term if we are influenced by experimental results to revise our initial view.

We may initiate such a process in a state in which we are completely neutral with respect to the question of whether a given response model term should be retained or rejected; that is, in a state in which we assume that the prior probability that the term is significant is 0.5 - just as likely to be real as not. This attitude is often represented as desirable in that it implies a laudable lack of prejudice with respect to the questions under investigation. And yet it does not seem illegitimate to assume that a subject matter expert would have a priori knowledge that ought to be taken into account. On the contrary, it seems unrealistic to assume otherwise. Bayesian inference provides a formal mechanism for combining a priori knowledge with new observations to generate revised insights, giving proper weight to the intrinsic uncertainty in what was originally believed and what has been newly learned.

The purpose of this paper has been simply to alert elements of the experimental aeronautics research community to a framework for making progress that may be new to them, and which the author has himself only recently begun to exploit. It is by no means a comprehensive tutorial on Bayesian inference, which is far too detailed a subject to cover adequately in a single conference paper and with which the author claims no substantial experience. Yet there seems to be a number of attractive elements to this approach to scientific inquiry, not the least of which from the author's perspective is its potential for seamless integration into the analytical methods of formal experiment design. Readers are encouraged to make their own independent inquiries and evaluations of the utility of Bayesian inference in experimental aeronautics research, but it is not unlikely that this topic will receive further attention as it becomes more familiar to researchers in our field.

\section{Acknowledgments}

This work was supported by the Aeronautics Test Program of the National Aeronautics and Space Administration.

\section{References}

${ }^{1}$ DeLoach, R., "Applications of Modern Experiment Design to Wind Tunnel Testing at NASA Langley Research Center," $A I A A-98-0713,36^{\text {th }}$ AIAA Aerospace Sciences Meeting and Exhibit. Reno, NV, January 1998.

${ }^{2}$ DeLoach, R. "Tailoring Wind Tunnel Data Volume Requirements Through the Formal Design Of Experiments," AIAA-982884, $20^{\text {th }}$ Advanced Measurement and Ground Testing Conference. Albuquerque, NM. June 1998.

${ }^{3}$ DeLoach, R., "Improved Quality in Aerospace Testing Through the Modern Design of Experiments (Invited)," AIAA 2000$0825,38^{\text {th }}$ AIAA Aerospace Sciences Meeting and Exhibit. Reno, NV. January 2000. 2007

${ }^{4}$ Box, G. E. P., and Draper, N., Response Surfaces, Mixtures, and Ridge Analyses, $2^{\text {nd }}$ Ed., John Wiley and Sons, New York,

${ }^{5}$ Montgomery, D. C., and Peck, E. A., Introduction to Linear Regression Analysis, $2{ }^{\text {nd }}$ Ed., John Wiley and Sons, New York, 1992.

${ }^{6}$ Myers, R. H., and Montgomery, D. C., Response Surface Methodology: Process and Product Optimization Using Designed Experiments, Wiley Series in Probability and Statistics, $2^{\text {nd }}$ Ed., John Wiley and Sons, New York, 2002.

${ }^{7}$ Draper, N. R., and Smith, H., Applied Regression Analysis, $3{ }^{\text {rd }}$ Ed., John Wiley and Sons, New York, 1998.

${ }^{8}$ Fisher, R. A., Statistical Methods and Scientific Inference, $2^{\text {nd }}$ Ed., Oliver and Boyd, London, 1959.

${ }^{9}$ Berger, J. O. Statistical Decision Theory and Bayesian Analysis. $2^{\text {nd }}$ Ed.. Springer Verlag, New York, 1999.

${ }^{10}$ Gelman, A., Carlin, B., Stern, H., and Rubin, D. B. Bayesian Data Analysis. ${ }^{\text {nd }}$ Ed.. Chapman \& Hall/CRD, Boca Raton, Florida, 2003.

${ }^{11}$ Bolstad, William M. Introduction to Bayesian Statistics, John Wiley and Sons, New York, 2004.

${ }^{12}$ Vassberg, J. C., Tinoco, E.N., Mani, M., Wahls, R. A., Morrison, J. H., Zickuhr,T., Laflin, K. R., Mavriplis, D. J., Brodersen, O. P., and Eisfeld, B., "Summary of the Third AIAA CFD Drag Prediction Workshop," Symposium on Computational Uncertainty, organized by the Applied Vehicle Technology Panel (AVT-147) of the North Atlantic Treaty Organization Research and Technology Agency. Athens, Greece, 3-6 December 2007.

${ }^{13}$ Quirk, J. J., "Computational Science: Same Old Silence, Same Old Mistakes, Something More is Needed," Adaptive Mesh Requirements-Theory and Applications, edited by T. Plewa, T. Linde, and V.G. Wiers, Springer-Verlag, 2004.

${ }^{14}$ Hemsch, M. J., and Morrison, J. H., "Statistical Analysis of the AIAA Drag Prediction Workshop CFD Solutions", Symposium on Computational Uncertainty, organized by the Applied Vehicle Technology Panel (AVT-147) of the North Atlantic Treaty Organization Research and Technology Agency. Athens, Greece, 3-6 December 2007.

${ }^{15}$ DeLoach, R., "Propagation of Computational Uncertainty Using the Modern Design of Experiments," Symposium on Computational Uncertainty, organized by the Applied Vehicle Technology Panel (AVT-147) of the North Atlantic Treaty Organization Research and Technology Agency. Athens, Greece, 3-6 December 2007.

${ }^{16}$ Ulbrich, N., and Volden, T., "Strain-Gage Balance Calibration Analysis Using Automatically Selected Math Models," AIAA 2005-4084, 41 ${ }^{\text {st }}$ AIAA/ASME/SAE/ASEE Joint Propulsion Conference and Exhibit, Tucson, Arizona, July 2005. 
${ }^{17}$ Ulbrich, N., and Volden, T., "Application of a New Calibration Analysis Process to the MK-III-C Balance," AIAA 2006-0517, 44 ${ }^{\text {th }}$ AIAA Aerospace Sciences Meeting and Exhibit, Reno, Nevada, January 2006.

${ }^{18}$ Ulbrich, N., and Volden, T., "A New Approach to Strain-Gage Balance Calibration Analysis," $5^{\text {th }}$ International Symposium on Strain-Gauge Balances, Aussois, France, May 2006.

${ }^{19}$ Ulbrich, N., and Volden, T., "Development of a New Software Tool for Balance Calibration Analysis," AIAA 2006-3434, $24^{\text {th }}$ AIAA Aerodynamic Measurement Technology and Ground Testing Conference, San Francisco, California, June 2006.

${ }^{20}$ Ulbrich, N., and Volden, T., "Analysis of Floor Balance Calibration Data using Automatically Generated Math Models," AIAA 2006-3437, 24 ${ }^{\text {th }}$ AIAA Aerodynamic Measurement Technology and Ground Testing Conference, San Francisco, California, June 2006.

${ }^{21}$ Parker, P. A., and Rhew R. D., "A Study of Automatic Balance Calibration System Capabilities," Second International Symposium on Strain-Gauge Balances, Bedford, England, UK, May 1999.

${ }^{22}$ Parker, P. A., and DeLoach, R., "Response Surface Methods for Force Balance Calibration Modeling," $19^{\text {th }}$ International Congress on Instrumentation in Aerospace Simulation Facilities, Cleveland, Ohio, August 2001.

${ }^{23}$ DeLoach, R., "Impact of Systematic Unexplained Variance on a Balance Calibration," 25 ${ }^{\text {th }}$ AIAA Aerodynamic Measurement Technology and Ground Testing Conference, San Francisco, California, June 2006.

${ }^{24}$ DeLoach, R., "Impact of Loading Selection and Sequencing on a Force Balance Calibration (Invited)," AIAA 2006-3436, 25th Aerodynamic Measurement Technology and Ground Testing Conference, San Francisco, California, June 2006.

${ }^{25}$ Parker, P .A., Morton, M., Draper, N., and Line, W., "A Single-Vector Force Calibration Method Featuring the Modern Design of Experiments," AIAA 2001-0170, 39 ${ }^{\text {th }}$ Aerospace Sciences Meeting and Exhibit, Reno, Nevada, January 2001.

${ }^{26}$ DeLoach, R., and Philipsen, I., "Stepwise Regression Analysis of MDOE Balance Calibration Data Acquired at DNW," AIAA 2007-0144, 45 ${ }^{\text {th }}$ Aerospace Sciences Meeting and Exhibit, Reno, Nevada, January 2007.

${ }^{27}$ Philipsen, I. and Zhai, J., "Comparative Study of Strain-Gauge Balance Calibration Procedures Using the Balance Calibration Machine," AIAA-2007-0143, 45 th Aerospace Sciences Meeting and Exhibit, Reno, Nevada, January 2007.

${ }^{28}$ Bayes, T. R. "An Essay Towards Solving a Problem in the Doctrine of Chances," Phil. Trans, Roy. Soc. London 53, 370, 1763. (reprinted in Biometrika (1958), 45, 293).

${ }^{29}$ Box, G. E. P., and Tiao, G. C., Bayesian Inference in Statistical Analysis, Wiley Classics Library Edition, John Wiley and Sons, New York, 1992. 